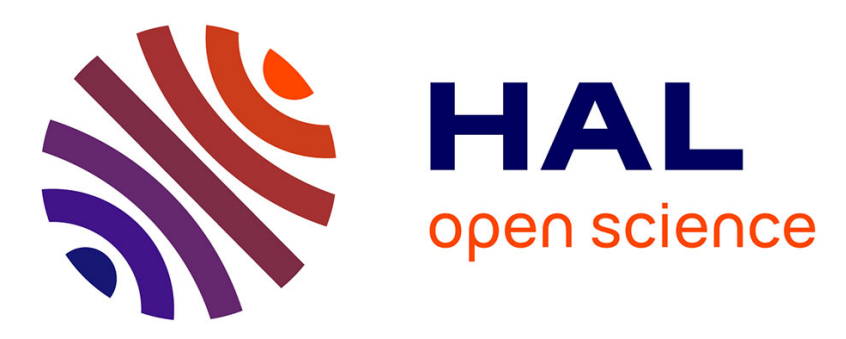

\title{
Sufficient Conditions for Global Integral Action via Incremental Forwarding for Input-Affine Nonlinear Systems
}

\author{
M Giaccagli, Daniele Astolfi, V Andrieu, L Marconi
}

\section{- To cite this version:}

M Giaccagli, Daniele Astolfi, V Andrieu, L Marconi. Sufficient Conditions for Global Integral Action via Incremental Forwarding for Input-Affine Nonlinear Systems. IEEE Transactions on Automatic Control, inPress, 10.1109/TAC.2021.3130876 . hal-03444503

\section{HAL Id: hal-03444503 \\ https://hal.science/hal-03444503}

Submitted on 23 Nov 2021

HAL is a multi-disciplinary open access archive for the deposit and dissemination of scientific research documents, whether they are published or not. The documents may come from teaching and research institutions in France or abroad, or from public or private research centers.
L'archive ouverte pluridisciplinaire HAL, est destinée au dépôt et à la diffusion de documents scientifiques de niveau recherche, publiés ou non, émanant des établissements d'enseignement et de recherche français ou étrangers, des laboratoires publics ou privés. 


\title{
Sufficient Conditions for Global Integral Action via Incremental Forwarding for Input-Affine Nonlinear Systems
}

\author{
Mattia Giaccagli and Daniele Astolfi and Vincent Andrieu and Lorenzo Marconi
}

\begin{abstract}
We study the problem of constant output regulation for a class of input-affine multi-input multi-output nonlinear systems, which don't necessarily admit a normal form. We allow the references and the disturbances to be arbitrarily large and the initial conditions of the system to range in the full-state space. We cast the problem in the contraction framework and we rely on the common approach of extending the system with an integral action processing the regulation error. We present then sufficient conditions for the design of a state-feedback control law able to make the resulting closed-loop system incrementally stable, uniformly with respect to the references and the disturbances. Such a property ensures uniqueness and attractiveness of an equilibrium on which output regulation is obtained. To this end, we develop an incremental version of the forwarding $\left(\bmod \left\{L_{g} V\right\}\right)$ approach. Finally, we provide a set of sufficient conditions for the design of a pure (smallgain) integral-feedback control. The proposed approach is also specialized for two classes of systems, that are linear systems having a Lipschitz nonlinearity and a class of minimum-phase systems whose zero-dynamics are incrementally stable.
\end{abstract}

Index Terms-Integral action, incremental stability, contraction theory, forwarding, nonlinear output regulation, minimumphase systems.

\section{INTRODUCTION}

This paper deals with the problem of constant output setpoint tracking and constant disturbances rejection for multiinput multi-output (MIMO) nonlinear systems. For linear systems, a common solution relies on the use of an integral action processing the regulation error. Such approach can be also seen as a particular case of the output regulation problem within which the so-called internal model principle has been developed (see, e.g., [9], [12]). For nonlinear systems, many solutions have been proposed based on integral action. With respect to the structural properties of the regulated system, the existing works can be mainly grouped in two main approaches.

In the first group of works, it is commonly supposed the existence of a change of coordinates that puts the system into a normal form, [17, Chapter 4]. Depending on the properties of the zero-dynamics, different control designs

M. Giaccagli, D. Astolfi and V. Andrieu are with Univ Lyon, Université Claude Bernard Lyon 1, CNRS, LAGEPP UMR 5007, 43 boulevard du 11 novembre 1918, F-69100, Villeurbanne, France name.surnameduniv-lyonl.fr.

L. Marconi is with CASY - DEI, University of Bologna, Italy lorenzo.marconi@unibo.it.

Authors acknowledge Université de Lyon 1 and Ecole Doctorale EEA for funding the $\mathrm{PhD}$ thesis of the first author. have been proposed: see, among others, [6], [19], [21] for single-input single-output (SISO) minimum-phase systems; [7], [44] for MIMO minimum-phase systems; [16] for SISO non-minimum phase systems. In these settings, output regulation can be ensured for arbitrarily large references and/or disturbances with a semi-global (or global) domain of attraction, provided that the zero-dynamics possess good uniform attractivity properties. However, such approaches cannot be followed when a global normal-form doesn't exist or its explicit expression is hard to be computed.

A second group of works approaches the problem in a "coordinate-free" framework, trying to develop a feedback law in the original coordinates. In this setting, the key observation is that when a system is augmented with an integral action processing the regulation error, the resulting extended system is in the so-called feedforward form, for which forwarding stabilization techniques can be applied [20], [27], [33]. Following this paradigm, if a given system can be globally stabilized at the origin, then a global stabilizing feedback-law for the unperturbed system (i.e., when disturbances and references are zero) extended with an integral action can be systematically designed under common controllability conditions, which are necessary and sufficient in the linear case, see [32]. Unfortunately, as shown in [5], [32], such an approach can be used to address the problem of output regulation with a domain of attraction which is semi-global in the initial conditions but only local in the amplitude of the references and disturbances. In other words, when the size of those signals is "large", the existence of a stable equilibrium is not guaranteed anymore.

By following this "coordinate-free" approach, the objective of this work is to develop a new approach to solve the problem of constant output regulation for MIMO nonlinear systems, with possibly more inputs that outputs, with a global domain of attraction and without restrictions on the amplitude of the set-point and of the disturbances. To this end, we focus on the class of input-affine systems with disturbances satisfying a matching condition, namely acting in the same directions of the control inputs, see, e.g., [34], [35]. We extend the system with an integral action processing the regulation error, and we look for state-feedback laws able to enforce some desired "good properties" for the resulting extended system. In particular, the goal is to obtain a closedloop system possessing an equilibrium that is globally attractive, uniformly in the disturbances and references, and at 
which the regulation objective is fulfilled. Such a property can be achieved if the system is convergent (see, e.g., [31]) or incrementally input-to-state stable (see, e.g., [4]). As a consequence, the objective of this article is to develop a new version of forwarding that incrementally stabilizes a system extended with an integral action. Furthermore, we show that such an incremental property is uniform in the disturbances and references if a Killing vector field (see, e.g., [28]) property is verified.

The contributions of this work are several. First, we develop a new set of sufficient conditions able to determine whether a system is incrementally stable, possibly uniformly in the initial conditions and in the disturbances. The conditions are based on a metric analysis. The cases of uniform and non-uniform contractions are considered and two new results are given in the Appendix. The first provides a set of sufficient conditions to establish the incremental stability properties of systems on an invariant compact set. The second proves the existence of a unique attractive equilibrium for non-uniform globally contractive systems. Then, we study the design of forwarding-based control laws able to incrementally stabilize a given system. Two different approaches are followed. First, we provide a new extension of the forwarding design with exact change of coordinates [27, Section IV] in the incremental context. This extends our preliminary results published in [14] for the SISO case. Then, in Section IV] we propose an incremental version of the forwarding $\bmod \left\{L_{g} V\right\}$ approach first appeared in [33]. Such result contribute to the number of existing techniques able to achieve incremental stability properties, such as backstepping [46], LMI [3], [8], and circle criterion [43], [45]. Finally, under more restrictive robustness-like conditions, we also propose a small-gain pure integral-feedback control law, providing a new set of sufficient conditions which are easier to verify and more constructive with respect to [39]. As a particular case of application, the proposed incremental forwarding designs are applied to the class of linear system matched with a Lipschitz nonlinearity and to the class of minimum-phase systems with incrementally stable zero-dynamics.

The rest of the paper is structured as follows. The problem statement and some preliminaries tools are given in Section II] The main results, concerning the incremental forwarding designs, are presented in Sections III and IV] Two study cases are analyzed in Section $\mathrm{V}-\mathrm{A}$ A review and some new results on contraction theory are included in the Appendix.

\section{A. Notations}

We define $\mathbb{R}_{>0}=(0, \infty)$ and $\mathbb{R}_{\geq 0}=[0, \infty)$. We denote with $|\cdot|$ the Euclidean norm and with $\|\cdot\|$ the standard induced matrix norm. Given an open set $\mathcal{O} \subset \mathbb{R}^{n}$, we denote with $\mathrm{Cl}(\mathcal{O})$ its closure. We denote with $I_{n}$ the Identity matrix of dimension $n \times n$. Given a vector field $f: \mathbb{R}^{n} \rightarrow \mathbb{R}^{n}$, we use the following compact notation $\nabla_{x} f(x):=\frac{\partial f}{\partial x}(x)$ or simply $\nabla f(x)$ when there is no confusion. Given a vector field $f: \mathbb{R}^{n} \rightarrow \mathbb{R}^{n}$ and a $C^{1}$ mapping $h: \mathbb{R}^{n} \rightarrow \mathbb{R}^{m}$, we denote the Lie derivative of $h$ along $f$ at $x$ as $L_{f} h(x):=$ $\langle\nabla h(x), f(x)\rangle=\frac{\partial h}{\partial x}(x) f(x)$. Given a 2-tensor $P: \mathbb{R}^{n} \rightarrow$ $\mathbb{R}^{n \times n}$ taking symmetric values and a vector field $f: \mathbb{R}^{n} \rightarrow$ $\mathbb{R}^{n}$, we denote the Lie derivative of the tensor $P$ along $f$ as $L_{f} P(x)$, defined as

$$
\begin{aligned}
L_{f} P(x) & :=\mathfrak{d}_{f} P(x)+P(x) \nabla f(x)+\nabla f^{\top}(x) P(x), \\
\mathfrak{d}_{f} P(x) & :=\lim _{t \rightarrow 0} \frac{P\left(X\left(x_{0}, t\right)\right)-P\left(x_{0}\right)}{t},
\end{aligned}
$$

where and $X\left(x_{0}, t\right)$ is the solution of

$$
\frac{\partial}{\partial t} X\left(x_{0}, t\right)=f\left(X\left(x_{0}, t\right)\right), \quad X\left(x_{0}, 0\right)=x_{0}, \quad \forall t \geq 0 .
$$

Given a 2-tensor $P: \mathbb{R}^{n} \rightarrow \mathbb{R}^{n \times n}$ and a $C^{1}$ vector field $g: \mathbb{R}^{n} \rightarrow \mathbb{R}^{n}$ (respectively a $C^{1}$ matrix function $G: \mathbb{R}^{n} \rightarrow$ $\mathbb{R}^{n \times m}$ ), we say that $g$ is a "Killing vector" (respectively, satisfy the "Killing matrix" property) for $P$, if $L_{g} P(x)=0$ (resp. $L_{G_{i}} P(x)=0$ for all $i=1, \ldots, m$, with $G_{i}$ denoting the $i$-th column of $G$ ).

\section{Problem Statement and Preliminaries}

\section{A. The Global (Integral) Output Regulation Problem}

This paper focuses on the constant global output regulation problem for nonlinear systems of the form

$$
\begin{aligned}
& \dot{x}=f(x)+g(x)[u+d] \\
& e=h(x)-r
\end{aligned}
$$

where $x \in \mathbb{R}^{n}$ is the system's state, $u \in \mathbb{R}^{m}$ is the control input, $e \in \mathbb{R}^{p}$ is the error between the output $h(x)$ to be regulated and a constant reference $r \in \mathbb{R}^{p}$, and $d \in \mathbb{R}^{m}$ is a constant unknown disturbance satisfying the so called "matching conditions" (see, e.g., [34], [35] and references therein), i.e., acting on the same directions of the controller $u$. We suppose that the functions $f: \mathbb{R}^{n} \mapsto \mathbb{R}^{n}, g: \mathbb{R}^{n} \mapsto$ $\mathbb{R}^{n \times m}$ and $h: \mathbb{R}^{n} \mapsto \mathbb{R}^{p}$ are $C^{1}$ with $m \geq p$. Furthermore $f$ and $h$ are zero at the origin.

The problem that is considered in this paper is the design of a state feedback control law for system (1) that is able to achieve output set-point tracking and disturbance rejection, that is, $\lim _{t \rightarrow \infty} e(t)=0$. Following [5], [32], and in the spirit of [9], [12], we focus on the implementation of a dynamic controller involving an integral action processing the error.

Specifically, we extend the system with a bunch of integrators of the form

$$
\dot{\eta}=e
$$

with $\eta \in \mathbb{R}^{p}$, and we look for a state feedback control law $\phi: \mathbb{R}^{n \times p} \mapsto \mathbb{R}^{m}$ of the form

$$
u=\phi(x, \eta)
$$

with $\phi(0,0)=0$ such that there exist two sets $\mathcal{S} \subseteq \mathbb{R}^{n+p}$ and $\mathcal{W} \subseteq \mathbb{R}^{m+p}$, containing their respective origins, such that the following properties are fulfilled:

1) if $(d, r)=(0,0)$, the origin of the closed loop system (1)-(2) is globally asymptotically stable;

2) for all constant $(d, r) \in \mathcal{W}$ and all initial conditions $\left(x_{0}, \eta_{0}\right) \in \mathcal{S}$, the closed loop system (1)-(2) has bounded trajectories and $\lim _{t \rightarrow+\infty} e(t)=0$. 
Then, we have the following problem definitions.

- If $\mathcal{S}$ and $\mathcal{W}$ are non-empty, the Regional (Integral) Output Regulation Problem is solved.

- If $\mathcal{S}=\mathbb{R}^{n+p}$ and $\mathcal{W}=\mathbb{R}^{m+p}$, the Global (Integral) Output Regulation problem is solved.

- If $\mathcal{S}$ is non-empty and $\mathcal{W}$ is of the form $\mathcal{W}=\emptyset \times \mathcal{R}$ for some non-empty set $\mathcal{R} \subseteq \mathbb{R}^{p}$, then the (Integral) Output Set-point Tracking Problem is solved.

Without the use of normal forms the construction of the control law $\phi$ is not straightforward, especially whenever the size of the disturbances is large in amplitude. For instance, the results presented in [5], [32] provide a solution that is semi-global in the amplitude of the state $(x, \eta)$ but only local in the size of $(d, r)$. One of the contributions of this work is to propose a set of sufficient conditions to obtain global results.

The crucial point in the synthesis of an integral controller is to ensure the existence of an equilibrium for every value of $(d, r)$. This amounts to asking that the flow admits a fixed point which can be obtained via Banach fixed point theorem ([22, Appendix B]) if the flow itself defines a contraction. To make the paper self contained, the Appendix provides some fundamental definitions and results related to contractions theory that are employed throughout the paper.

\section{B. From Contraction to Regulation}

System (1)-(2) leads to a closed-loop system of the form

$$
\dot{\mathcal{X}}=\varphi(\mathcal{X})+\Gamma(\mathcal{X}) w
$$

where $\mathcal{X}=(x, \eta) \in \mathbb{R}^{n+p}, w=(d, r) \in \mathbb{R}^{m+p}$, and the functions $\varphi$ and $\Gamma$ are defined as

$$
\begin{gathered}
\varphi(\mathcal{X}):=\left(\begin{array}{c}
f(x)+g(x) \phi(x, \eta) \\
h(x)
\end{array}\right), \\
\Gamma(\mathcal{X}):=\left(\begin{array}{ll}
\Gamma_{d}(\mathcal{X}) & \Gamma_{r}
\end{array}\right), \Gamma_{d}(\mathcal{X}):=\left(\begin{array}{c}
g(x) \\
0
\end{array}\right), \Gamma_{r}:=\left(\begin{array}{c}
0 \\
-I_{p}
\end{array}\right) .
\end{gathered}
$$

Given any initial condition $\mathcal{X}_{0}$ in $\mathbb{R}^{n+p}$ and $w \in \mathbb{R}^{m+p}$, the corresponding solution of system (3) is denoted with $\mathcal{X}\left(\mathcal{X}_{0}, t, w\right)$.

The control law (2b) solves the (integral) output regulation problem if, for any value of $w \in \mathcal{W}$, there exists a unique equilibrium $\mathcal{X}^{*}=\left(x^{\star}, \eta^{\star}\right)$ that is globally asymptotically stable. Indeed, thanks to the integral action, at the equilibrium we have $\dot{\eta}=h\left(x^{\star}\right)-r=0$ and consequently the error $e$ converges asymptotically to zero along any solution of the closed loop system. Contraction theory can be used to solve the problem at hand (see, e.g., [31]), as highlighted in the following theorem.

Theorem 1. Given $f, g, h$, suppose there exist a $C^{1}$ function $\phi: \mathbb{R}^{n \times p} \mapsto \mathbb{R}^{m}$, a $C^{1}$ function $\mathcal{P}: \mathbb{R}^{n+p} \rightarrow \mathbb{R}^{(n+p) \times(n+p)}$ taking symmetric positive definite values, a $C^{0}$ function $\mathfrak{q}$ : $\mathbb{R}^{n+p} \rightarrow \mathbb{R}_{>0}$ taking positive values and two positive real numbers $\overline{\mathfrak{p}}, \mathfrak{p}>0$ such that the vector fields $\varphi, \Gamma$ defined in (4)-(5) satisfy the following set of properties

$$
\begin{array}{r}
\underline{\mathfrak{p}} I_{n+p} \leq \mathcal{P}(\mathcal{X}) \leq \overline{\mathfrak{p}} I_{n+p} \\
L_{\varphi} \mathcal{P}(\mathcal{X}) \leq-\mathfrak{q}(\mathcal{X}) I_{n+p} \\
L_{\Gamma_{r}} \mathcal{P}(\mathcal{X})=0 \\
L_{\Gamma_{d}} \mathcal{P}(\mathcal{X})=0
\end{array}
$$

for all $\mathcal{X} \in \mathbb{R}^{n+p}$. Then, the following holds.

1) If there exists a positive real number $\mathfrak{q}>0$ such that $\mathfrak{q}(\mathcal{X}) \geq \mathfrak{q}$ for all $\mathcal{X} \in \mathbb{R}^{n+p}$, then the Global (Integral) Output Regulation Problem is solved.

2) Otherwise, we have the following properties.

(a) Let $\mathcal{W}$ be a non-empty subset of $\mathbb{R}^{m+p}$ such that, for all $w$ in $\mathcal{W}$, there exists an initial condition $\mathcal{X}_{0} \in$ $\mathbb{R}^{n+p}$ such that the corresponding closed-loop system trajectory $\mathcal{X}\left(\mathcal{X}_{0}, w, t\right)$ is bounded. Then, the Regional (Integral) Output Regulation Problem is solved for $\mathcal{S}=\mathbb{R}^{n+p}$ and such a $\mathcal{W}$.

(b) There exists $\bar{w}>0$ such that, the Regional (Integral) Output Regulation Problem is solved for $\mathcal{S}=\mathbb{R}^{n+p}$ and $\mathcal{W}=\left\{w \in \mathbb{R}^{m+p},|w| \leq \bar{w}\right\}$.

Furthermore, if the condition (6d) is not verified, then, the above results 1) and 2) hold for the (Integral) Output Setpoint Tracking Problem, in particular with $\mathcal{W}$ of the form $\mathcal{W}=\emptyset \times \mathcal{R}$, for some $\mathcal{R} \subseteq \mathbb{R}^{p}$.

The statement of Theorem 1 involves many different results based on a contraction analysis of the vector field $\varphi$ of the closed-loop system (3) and how perturbations and references $w$ affect the system through the vector $\Gamma$.

First, conditions 6a and (6b), require the unforced closedloop system (3), i,e, for $w=0$, to be globally contractive, but possibly non-uniformly with respect to the full-state space $\mathcal{X}$. In other words, since $\mathfrak{q}$ can be non-uniformly lowerbounded, we cannot conclude that the unforced closed-loop system is $\delta \mathrm{ES}$ (See Definition 1 in the Appendix). However, since $\varphi(0)=0$, the origin is globally asymptotically stable (see Proposition 8 in the Appendix). If the contraction is uniform (see item 1), that is $\mathfrak{q}(x) \geq \mathfrak{q}>0$, and the Killing vector conditions (6c), (6d), with respect to $\Gamma$ are verified, then, the system is still contractive for any $\mathcal{X}$ and $w$. In this case, the key role of the Killing vector property is to ensure the distance among different trajectories to be invariant with respect to the amplitude of external signals. In turn, such a geometric condition ensures that the contractivity property of system (3) are invariant with respect to $w$, and the convergence to an equilibrium is guaranteed. Note that such a Killing property is always verified when $\Gamma$ is a linear mapping and $P$ is a constant matrix. This is the case, for instance, of linear systems.

As we shall see in the sequel, the existence of a feedbacklaw (2b) ensuring a uniform contraction is not always possible. However, under milder assumptions, we shall see that it is in general possible to obtain a non-uniform contraction, according to the conditions of item 2 . In such a case, output regulation is obtained globally in the initial conditions, but 
only regionally with respect to the exosignal $w$, that is, only for $w \in \mathcal{W}$ for some compact set $\mathcal{W}$. Item 2 (a) and (b) provide two different conditions for the estimation of such a set $\mathcal{W}$. It is of particular interest the first set of conditions (a), in which it suffices to verify whether the resulting trajectory is bounded forward in time to conclude asymptotic regulation. Nonetheless, the condition (b) ensures that the set $\mathcal{W}$ is non-empty. In other words, asymptotic regulation is always guaranteed globally in the state $\mathcal{X}$ and at least locally in the size of the exosignal $w$. Note that such a result is also new with respect to [5], where output regulation is obtained only semi-globally in $\mathcal{X}$ and locally in $w$.

Finally, since the existence of a metric $\mathcal{P}$ which is of Killing for both vectors $\Gamma_{r}$ and $\Gamma_{d}$ is not always possible, the last part of the theorem states that whenever only $\Gamma_{r}$ is a Killing vector for $\mathcal{P}$, then, all previous arguments hold by considering only the case of tracking problem, i.e. with $d=0$. As a matter of fact, as we shall see in the subsequent sections, it is easier to design a feedback law satisfying the condition (6c) than (6d), because $\Gamma_{r}$ is constant while $\Gamma_{d}$ is not.

Proof of Theorem 1 . First, note that the origin of (3) for $w=0$ is an equilibrium. Hence, direct application of Proposition 8 implies that the equilibrium is globally attractive when $w=0$. Furthermore, it is also locally exponentially stable. This can be proved by using the first order approximation of system (3) at the origin and the Lyapunov function $V(\mathcal{X})=\mathcal{X}^{\top} \mathcal{P}(0) \mathcal{X}$, and inequality $(6 \mathrm{~b})$, which is $L_{\varphi} \mathcal{P}(0) \leq-\mathfrak{q}(0) I<0$ at the origin. This establish the first property 1). Concerning the second property, we will now prove the two items separately.

Item 1 . Since $\mathfrak{q}(\mathcal{X}) \geq \mathfrak{q}>0$, the inequality $6 \mathrm{~b}$ is strict for all $\mathcal{X} \in \mathbb{R}^{n+p}$. Furthermore, by using the the Killing matrix properties (6c), (6d), we have

$$
\begin{aligned}
L_{\varphi+\Gamma w} \mathcal{P}(\mathcal{X}) & =L_{\varphi} \mathcal{P}(\mathcal{X})+L_{\Gamma} \mathcal{P}(\mathcal{X}) w \\
& =L_{\varphi} \mathcal{P}(\mathcal{X}) \\
& \leq-\underline{q} I_{n+p},
\end{aligned}
$$

for all $\mathcal{X} \in \mathbb{R}^{n+p}$ and all $w \in \mathbb{R}^{m+p}$. As a consequence, system (3) is an autonomous system satisfying the conditions of Theorem 2 in the Appendix. We deduce that system (3) is $\delta$ GES. Direct application of Lemma 2 in the Appendix ensures that, for any $w$, there exists a unique equilibrium point $\mathcal{X}^{\star}=\left(x^{\star}, \eta^{\star}\right)$ which exponentially attracts all solutions. On such equilibrium, we obtain $\dot{\eta}=0$ and hence $e=0$, concluding the proof of the statement.

Item $2(a)$. Let $w$ be in $\mathcal{W}$ and let $\mathcal{X}_{0} \in \mathbb{R}^{n+p}$ such that $\mathcal{X}\left(\mathcal{X}_{0}, w, t\right)$ is bounded. Let $\mathcal{C} \subset \mathbb{R}^{n+p}$ be defined as

$$
\mathcal{C}:=\mathrm{Cl}\left\{\bigcup_{t \geq 0}^{\infty} \mathcal{X}\left(\mathcal{X}_{0}, w, t\right)\right\}
$$

and note that $\mathcal{C}$ is a forward invariant compact set, in view of [41, Lemma 6.4]. Let $\underline{\mathfrak{q}}_{\mathcal{O}}:=\min _{\{(x, \eta) \in \mathcal{O}\}} \mathfrak{q}(x, \eta)>0$ where $\mathcal{O}$ is the set defined as

$$
\mathcal{O}:=\left\{\mathcal{X} \in \mathbb{R}^{n+p}, \max _{s \in \mathcal{C}}|\mathcal{X}-s|<\mathfrak{r}\right\},
$$

for some positive real number $\mathfrak{r}$ satisfying

$$
\mathfrak{r}>\frac{\sqrt{\mathfrak{p}}}{2 \sqrt{\mathfrak{p}}} \max _{\left(\mathcal{X}_{1}, \mathcal{X}_{2}\right) \in \mathcal{C}^{2}}\left|\mathcal{X}_{1}-\mathcal{X}_{2}\right| .
$$

Keeping in mind that $\Gamma_{d}, \Gamma_{r}$ satisfy the Killing matrix properties 6c , 6d for $\mathcal{P}$, by using computations similar to (7), we obtain $L_{\varphi+\Gamma w} \mathcal{P}(\mathcal{X}) \leq-\underline{q}_{\mathcal{O}} I_{n+p}$ for all $(x, w) \in \mathcal{O} \times \mathcal{W}$. Hence Proposition 7 applies (see Appendix) and therefore system (3) is $\delta \mathrm{ES}$ in $\mathcal{C}$. Then, Lemma 2 in Appendix implies the existence of an equilibrium $\mathcal{X}^{\star} \in \mathcal{C}$. From proposition 8 it yields that this equilibrium is unique and it is globally attractive. Again, on such an equilibrium, we have $\dot{\eta}=0$, and hence $e=0$.

Item $2(b)$. We already proved that the equilibrium of $(3)$ is locally exponentially stable for $w=0$. Hence, by [5, Lemma 5], there exists $\bar{w}>0$ such that, for all $w \in \mathcal{W}, \mathcal{W}:=$ $\left\{w \in \mathbb{R}^{m+p}:|w| \leq \bar{w}\right\}$, system (3) admits an equilibrium $\mathcal{X}^{*} \in \mathbb{R}^{n+p}$ which is locally exponentially stable. Hence, employing Item 2 (a) with this set $\mathcal{W}$, the result follows.

Motivated by the conditions of Theorem 1, in the rest of the paper we will design a control law able to guarantee both the contraction of $\varphi$ for some metric $\mathcal{P}$, and the Killing matrix property with respect to $\Gamma$. To this end, we will develop an incremental version of the forwarding approach, which is briefly recalled in the next section.

\section{Highlights on Forwarding Design}

When $(d, r)=(0,0)$, system (3) reads

$$
\begin{aligned}
\dot{x} & =f(x)+g(x) u, \\
\dot{\eta} & =h(x),
\end{aligned}
$$

where $x \in \mathbb{R}^{n}, u \in \mathbb{R}^{m}, \eta \in \mathbb{R}^{p}$. Systems in the form (8), are said to be in the so-called feed-forward form (see, e.g., [27]). Many techniques have been developed in the past years for systematic stabilization of this class of systems. Among them, we recall below the so called forwarding approach (see, for instance, [5], [20], [32], [33], [38]).

The forwarding approach is based on the assumption that the subsystem 8a is stabilizable by a state-feedback law. Following [27], the following result is obtained (see [27, Lemma IV.2]).

Lemma 1. Assume there exists a $C^{1}$ functions $\phi_{0}: \mathbb{R}^{n} \mapsto$ $\mathbb{R}^{m}$ such that the origin of

$$
\dot{x}=f_{0}(x), f_{0}(x):=f(x)+g(x) \phi_{0}(x),
$$

is Locally Exponentially Stable and Globally Asymptotically Stable. Then, there exists a $C^{2}$ function $M: \mathbb{R}^{n} \rightarrow \mathbb{R}^{p}$ solution of

$$
L_{f_{0}} M(x)=h(x) .
$$

Two typical control laws can be given via forwarding approaches.

- The first one is in the form

$$
u=\phi_{0}(x)-\left[L_{g} V(x)-L_{g} M(x)^{\top}(\eta-M(x))\right],
$$


where $V$ is the Lyapunov function associated to the feedback $\phi_{0}$, i.e. such that $L_{f_{0}} V(x)<0$ for all $x \neq 0$.

- The second one follows the small control strategy

$$
u=\phi_{0}(x)+\ell(x) L_{g} M(x)^{\top}(\eta-M(x)),
$$

where $\ell: \mathbb{R}^{n} \rightarrow \mathbb{R}_{>0}$ is a sufficiently small function (needed to compensate the absence of the term $L_{g} V$ ). The control law (12) can be used, for instance, when the Lyapunov function $V$ is unknown.

See also [5, Section III] or [32] and references therein for more details. We have the following result.

Proposition 1 (Forwarding design). Let the conditions of Lemma 1 hold. Furthermore, suppose that the function $M$ defined in (10) satisfies

$$
\operatorname{rank}\left(L_{g} M(0)\right)=p .
$$

Then, there exists $\ell: \mathbb{R}^{n} \rightarrow \mathbb{R}_{>0}$ such that the origin of system (8) in closed-loop with (12) is Globally Asymptotically Stable and Locally Exponentially Stable.

Condition (13) is guaranteed if system (8a) with output $y=h(x)$ is locally controllable at the origin. Such property, also known as non-resonance condition in regulation theory [18. Chapter 1.4], corresponds to the condition $C A^{-1} B$ full rank, where $A:=\nabla f(0), B:=g(0), C:=\nabla h(0)$. See also [5] for more details. Although the existence of $M$ is always guaranteed under the conditions of Lemma 11, its explicit expression may not be always easy to find, since it involves the solution of the partial differential equation (10). As a consequence, in order to provide additional degrees of freedom for the design of the control law (12), several extensions have been developed through the years. See, for instance, [5, Section III.B].

A particular design of interest for our work is the design proposed in [33] which extends the control laws of the form (11), and denoted as "forwarding $\bmod \left\{L_{g} V\right\}$ ", and highlighted in the following proposition. We recall that such design has been proposed for single-input single-output systems $(m=p=1)$.

Proposition 2 (Forwarding $\bmod \left\{L_{g} V\right\}$ ). Consider system 9) and suppose to know a positive definite Lyapunov function $V: \mathbb{R}^{n} \rightarrow \mathbb{R}$ satisfying $L_{f_{0}} V(x)<0$ for all $x \neq 0$. Assume, moreover, to know a $C^{1}$ function $\bar{M}: \mathbb{R}^{n} \mapsto \mathbb{R}$ with $\bar{M}(0)=$ 0 and $a C^{0}$ function $k(x)$ such that the following holds.

(i) $L_{f_{0}} \bar{M}(x)=h(x)+k(x) L_{g} V(x)$.

(ii) $L_{g} \bar{M}(0) \neq 0$.

(iii) For all $\left\{x \in \mathbb{R}^{n}: L_{g} \bar{M}(x) \neq 0, x \neq 0\right\}$, then

$$
L_{f_{0}} \bar{M}(x)-\frac{k(x)\left(L_{g} V\right)^{2}(x)}{L_{g} \bar{M}(x)}<0 .
$$

Then, the origin of (8) in closed-loop with

$$
\begin{aligned}
u=\phi_{0}(x) & -\frac{k(x) L_{g} V(x)}{L_{g} \bar{M}(x)} \\
& -\left[L_{g} V(x)-L_{g} \bar{M}(x)(\eta-\bar{M}(x))\right],
\end{aligned}
$$

is Globally Asymptotically Stable and Locally Exponentially Stable.
Proof. See [33, Proposition 1].

The interest of the condition (i) in Proposition 2 concerns the computation of $\bar{M}$, which can be interpreted as an approximated version of the solution of the PDE (10), in which the mismatch between the exact solution $M$ and its approximated version $\bar{M}$ is represented by the term $k(x) L_{g} V(x)$. Such an approximated solution is introduced to add extra degrees of freedom when the exact solution of $M$ is hard to obtain. Such a mismatch term has no destabilizing effect in light of the item (iii) of the proposition. The reader may refer to [33] for a more detailed discussion.

As shown in [5], global stabilization of the origin implies that the output regulation problem is solved semi-globally with respect to the state but only locally with respect to the size of the exosignals. In order to weaken this restriction, we aim at ensuring contractivity properties for the closed-loop systems. The goal of Section III is therefore to develop an incremental version of the forwarding design satisfying the conditions of Theorem 1

\section{INCREMENTAL FORWARDING}

\section{A. Assumptions}

In this section we state the main assumptions for system (1) which are needed in order to design a feedback-law of the form (2) that is able to satisfy the conditions of Theorem 1 For this, we follow a forwarding-like approach, as highlighted in Section II-C. In our contraction framework, we assume the existence of a pre-stabilizing feedback ensuring a uniform contraction according to a metric satisfying the Killing vector property with respect to the function $g$.

Assumption 1 (Contractivity and Killing Vector). Consider system (1) with the function $g$ fulfilling $\|g(x)\| \leq \bar{g}$ for some positive $\bar{g}>0$ for all $x \in \mathbb{R}^{n}$. There exist a known $C^{1}$ function $\phi_{0}: \mathbb{R}^{n} \mapsto \mathbb{R}^{m}, \phi_{0}(0)=0$, a $C^{1}$ function $P: \mathbb{R}^{n} \rightarrow \mathbb{R}^{n \times n}$ taking symmetric positive definite values $P(x)=P(x)^{\top}>0$ for all $x \in \mathbb{R}^{n}$, and real numbers $p, \bar{p}, \lambda_{0}>0$ such that, by denoting

$$
\dot{x}=f_{0}(x), \quad f_{0}(x):=f(x)+g(x) \phi_{0}(x),
$$

the following properties hold for all $x \in \mathbb{R}^{n}$

$$
\begin{gathered}
\underline{p} I_{n} \leq P(x) \leq \bar{p} I_{n}, \quad L_{f_{0}} P(x) \leq-2 \lambda_{0} I_{n}, \\
L_{g} P(x)=0 .
\end{gathered}
$$

According to Theorem 2 (see Appendix), the feedback law $u=\phi_{0}(x)$ ensures the vector field $f_{0}$ to be $\delta$ GES with respect to the metric $P$. The computation of $\phi_{0}$ can be obtained, for instance, by following the design techniques in [31. Chapter 5], or [2], [3], [13], [29], [46]. Furthermore, in (17), we ask that the (Riemaniann) metric induced by $P$ is invariant along $g$, i.e., $g$ is a Killing vector for $P$. For instance, in the case $g$ is constant, the property (17) is satisfied for any constant metric $P$. Indeed, as we allow disturbances to have any amplitude, their contribution to the system trajectory must be always zero, that is, the contractivity property of the autonomous system is not affected at all by $d$. This means 
that the system preserves its contractivity properties in the directions provided by $g$, namely, in the directions in which the control law and the perturbations $d$ act. In other words, it is possible to show that Assumption 1 implies system

$$
\dot{x}=f_{0}(x)+g(x) d
$$

to be incrementally input-to-state-stable ${ }^{1}$ with respect to $d$. See also Section III.A in [25] for further details on condition (17). The Killing vector property can be obtained, for instance, by means of computational tools (see [42]).

Since system (15) is $\delta$ GES and the origin is an equilibrium, it is also globally exponentially stable. As a consequence, by applying Lemma 1, we know the existence of a function $M: \mathbb{R}^{n} \mapsto \mathbb{R}^{p}$ satisfying (10). Similarly to the condition (13) of Proposition 1, we suppose then that $L_{g} M(x)$ satisfies a controllability-like condition, which, in our incremental framework, is stated as follows.

Assumption 2 (Controllability along trajectories). There exists a positive real number $\alpha>0$ satisfying

$$
L_{g} M(x) L_{g} M^{\top}(x) \geq \alpha I_{p} \quad \forall x \in \mathbb{R}^{n}
$$

where $M$ is the solution of (10).

Assumption 2 can be read as a uniform controllability-like assumption for extended dynamics

$$
\dot{x}=f_{0}(x)+g(x) u \quad \dot{\eta}=h(x)
$$

corresponding to the "incremental version" of condition (13).

Remark 1. Note that for a linear system of the form

$$
\dot{x}=A x+B u, \quad \dot{\eta}=C x
$$

Assumptions 1 and 2 boil down to ask, respectively, for $(A, B)$ to be stabilizable (in this case the metric $P$ is constant and (17) is automatically satisfied) and the non-resonance condition $\operatorname{rank}\left(C A^{-1} B\right)=p$ to hold. In linear output regulation theory (see, e.g. [9], [12], or [18, Chapter 1]) these conditions are necessary and sufficient to solve the Global Output Regulation Problem.

\section{B. Design for Output Regulation Problem}

Under the assumptions stated in Section III-A we are now in the position to state the first result, concerning the design of a forwarding-based control law for system (11) satisfying the hypothesis (6a) (6b) and (6c) of Theorem 11. Taking inspiration from (12), we focus on a control law of the form

$$
\phi(x, \eta)=\phi_{0}(x)+\kappa \Psi(x) \beta(\eta-M(x)),
$$

where the function $\Psi: \mathbb{R}^{n} \rightarrow \mathbb{R}^{m \times p}$ is defined as

$$
\Psi(x):=L_{g} M(x)^{\top}\left(L_{g} M(x) L_{g} M(x)^{\top}\right)^{-1},
$$

$\kappa \in \mathbb{R}$ is a control gain parameter and $\beta: \mathbb{R}^{p} \rightarrow \mathbb{R}^{p}$ is a $C^{1}$ function to be specified. We have the following result.

${ }^{1}$ See, for instance, [4].
Proposition 3 (Incremental (non-uniform) forwarding). Consider system (1) and suppose Assumptions 1 and 2 hold. Suppose, moreover, that there exist positive real numbers $L_{M}>0$ and $k_{1} \geq 0$ such that the following inequalities are verified

$$
\begin{aligned}
\|\nabla M(x)\| & \leq L_{M}, \\
\left\|\nabla_{x}(\Psi(x) v)\right\| & \leq k_{1}|v|, \quad \forall v \in \mathbb{R}^{p}
\end{aligned}
$$

for all $x \in \mathbb{R}^{n}$, with $\Psi$ defined as in (20) and with $M$ defined as in (10). Finally, select $\beta: \mathbb{R}^{p} \rightarrow \mathbb{R}^{p}$ as any $C^{1}$ function satisfying

$$
\|\beta(s)\| \leq \frac{1}{k_{1}}, \quad \nabla \beta(s)=\nabla \beta^{\top}(s), \quad 0<\nabla \beta(s) \leq I_{p},
$$

for all $s \in \mathbb{R}^{p}$. Then, there exists a positive real number $\bar{\kappa}>0$ such that, for any $\kappa \in(0, \bar{\kappa}]$, the closed-loop system (11)-(2), with $\phi$ selected as in (19), satisfies the properties (6a), (6b), and (6c) and the conditions of item 2 of Theorem 1 .

Remark 2. For instance, when $p=1$, a simple choice of $\beta$ satisfying the conditions in 23) is $\beta(s)=\mu \operatorname{atan}(s)$ with $\mu>0$ to be selected sufficiently small.

A direct consequence of Proposition 3 and Theorem 1 is the following corollary. The proof is omitted for space reasons.

Corollary 1. Under the assumptions of Proposition 3, the control law (2), with $\phi$ selected as in (19), satisfies the Regional (Integral) Output Tracking Problem for system (1) for some non-empty $\mathcal{R} \subset \mathbb{R}^{p}$, according to the statement of item 2 of Theorem 1

Proof of Proposition 3. Since the condition (6d) is not verified, in the rest of the proof we refer to the last part of the statement of Theorem 1 and we fix $d=0$. Now, consider then the following change of coordinates

$$
(x, \eta) \mapsto \chi:=(x, z), \quad z:=\eta-M(x),
$$

and recall that in view of Assumption 2 and the definition of $\Psi$ in $\left[20\right.$, we have $L_{g} M(x) \Psi(x)=I_{p}$ for all $x \in \mathbb{R}^{p}$. As a consequence, in the $\chi$-coordinates, the closed-loop system (1), (2), (19), with $d=0$ and any $r$, reads

$$
\dot{\chi}=F(\chi)+\Gamma_{r} r
$$

with $F$ defined as

$$
F(\chi):=\left(\begin{array}{c}
f_{0}(x)+\kappa g(x) \Psi(x) \beta(z) \\
-\kappa \beta(z)
\end{array}\right)
$$

and $\Gamma_{r}$ defined as in (5). Now, by recalling the triangular structure of $F$, the Jacobian $J_{F}$ of $F$ is given by

$$
J_{F}(\chi):=\nabla F(\chi)=\left(\begin{array}{cc}
J_{11}(\chi) & J_{12}(\chi) \\
0 & J_{22}(\chi)
\end{array}\right)
$$

where the components $J_{11}, J_{12}$ and $J_{22}$ are defined as

$$
\begin{aligned}
& J_{11}(\chi):=\nabla f_{0}(x)+\kappa \nabla(g(x) \Psi(x)) \beta(z), \\
& J_{12}(\chi):=\kappa g(x) \Psi(x) \nabla \beta(z), \quad J_{22}(\chi):=-\kappa \nabla \beta(z) .
\end{aligned}
$$


Consider a matrix valued function $\overline{\mathcal{P}}: \mathbb{R}^{n+p} \rightarrow$ $\mathbb{R}^{(n+p) \times(n+p)}$ of the form

$$
\overline{\mathcal{P}}(x):=\left(\begin{array}{cc}
P(x) & 0 \\
0 & \mathfrak{b} I_{p}
\end{array}\right),
$$

where $P$ is given by Assumption 1 and $\mathfrak{b}>0$ is some positive real number to be defined yet. By construction, the function $\overline{\mathcal{P}}$ takes symmetric positive values. Then, let also the function $\mathcal{L}: \mathbb{R}^{n+p} \rightarrow \mathbb{R}^{(n+p) \times(n+p)}$ be defined as

$$
\mathcal{L}(\chi):=L_{F} \overline{\mathcal{P}}(\chi)+\left(\begin{array}{cc}
\lambda_{0} I_{n} & 0 \\
0 & \kappa \nabla \beta(z)
\end{array}\right)
$$

with $\lambda_{0}$ given by Assumption 11 Observe that $\mathcal{L}$ takes symmetric values. We want to show now that $\mathcal{L}$ takes only negative definite values if $\kappa$ is selected small enough. To this end, let us decompose $\mathcal{L}$ as follows

$$
\mathcal{L}(\chi):=\left(\begin{array}{ll}
\mathcal{L}_{11}(\chi) & \mathcal{L}_{12}(\chi) \\
\mathcal{L}_{12}^{\top}(\chi) & \mathcal{L}_{22}(\chi)
\end{array}\right)
$$

By inspecting its components, by using the definition of $J_{F}$ and the Killing vector property $L_{g} P(x)=0$, see (17) in Assumption 1] we have

$$
\begin{aligned}
\mathcal{L}_{11}(\chi):= & L_{f_{0}} P(x)+\lambda_{0} I_{n}+\kappa P(x) g(x) \nabla \Psi(x) \beta(z) \\
& +\kappa(P(x) g(x) \nabla \Psi(x) \beta(z))^{\top}, \\
\mathcal{L}_{12}(\chi):= & \kappa P(x) g(x) \Psi(x) \nabla \beta(z), \\
\mathcal{L}_{22}(\chi):= & -\kappa(2 \mathfrak{b}-1) \nabla \beta(z) .
\end{aligned}
$$

By using $(23)$, we have $\mathcal{L}_{22}(\chi)<0$ for all $\chi \in \mathbb{R}^{n+p}$ and for any $\mathfrak{b}>\frac{1}{2}$. Furthermore, it is invertible for all $\chi \in \mathbb{R}^{n+p}$. As a consequence, $\mathcal{L}(\chi)$ is negative definite for all $\chi \in \mathbb{R}^{n+p}$ if its Schur complement, denoted as $\operatorname{SC}_{\mathcal{L}}(\chi)$, is also negative definite for all $\chi \in \mathbb{R}^{n+p}$. Now, by using the fact that $L_{f_{0}} P$ satisfies inequality (16), and that the functions $P, g, \beta$ and the derivative of $\Psi$ are bounded, see [16, 177, 21], and (23), it yields

$$
\mathcal{L}_{11}(\chi) \leq-\left(\lambda_{0}-2 \kappa \bar{p} \bar{g}\right) I_{n} \quad \forall \chi \in \mathbb{R}^{n+p} .
$$

Moreover, by combining inequalities (18) and 21) and by recalling the definition of $\Psi$ in 20$]$, we also obtain

$$
\|\Psi(x)\| \leq \frac{L_{M} \bar{g}}{\alpha}
$$

By combining all previous bounds together, we obtain

$$
\begin{aligned}
\mathrm{SC}_{\mathcal{L}}(\chi):= & \mathcal{L}_{11}(\chi)-\mathcal{L}_{12}(\chi) \mathcal{L}_{22}(\chi)^{-1} \mathcal{L}_{12}(\chi)^{\top} \\
\leq & -\left(\lambda_{0}-2 \kappa \bar{p} \bar{g}\right) I_{n} \\
& +\frac{\kappa}{2 \mathfrak{b}-1} P(x) g(x) \Psi(x) \nabla \beta(z) \Psi^{\top}(x) g^{\top}(x) P(x) \\
\leq & -\left(\lambda_{0}-\kappa \bar{p} \bar{g}\left(2+\frac{\bar{p} L_{M}^{2} \bar{g}^{3}}{2(2 \mathfrak{b}-1) \alpha^{2}}\right)\right) I_{n} .
\end{aligned}
$$

Hence, with $\mathfrak{b}>\frac{1}{2}$, selecting

$$
\bar{\kappa}=\lambda_{0}\left(\bar{p} \bar{g}\left(2+\frac{\bar{p} \bar{g}^{3} L_{M}^{2}}{2(2 \mathfrak{b}-1) \alpha^{2}}\right)\right)^{-1},
$$

it implies that $\mathrm{SC}_{\mathcal{L}}(\chi) \leq 0$ for all $\chi \in \mathbb{R}^{n+p}$ and therefore $\mathcal{L}(\chi) \leq 0$ for all $\kappa \in(0, \bar{\kappa}]$ and for all $\chi \in \mathbb{R}^{n+p}$. Consequently, recalling the definition of $\mathcal{L}$ in 27], it yields

$$
L_{F} \overline{\mathcal{P}}(\chi) \leq-\min \left\{\lambda_{0}, \kappa \nabla \beta(z)\right\} I_{n+p} .
$$

Note that the metric $\overline{\mathcal{P}}$ has been obtained in the $\chi$ coordinates. In order to complete the proof, we need to come back into the original coordinates $\mathcal{X}=(x, \eta)$. In particular, the metric $\mathcal{P}$ is defined as

$$
\mathcal{P}(\chi):=E(x)^{\top} \overline{\mathcal{P}}(\mathcal{X}) E(x), E(x):=\left(\begin{array}{cc}
I_{n} & 0 \\
-\nabla M(x) & I_{p}
\end{array}\right),
$$

giving

$$
\mathcal{P}(\chi)=\left(\begin{array}{cc}
P(x)+\mathfrak{b} \nabla M^{\top}(x) \nabla M(x) & -\mathfrak{b} \nabla M^{\top}(x) \\
-\mathfrak{b} \nabla M(x) & \mathfrak{b} I_{p}
\end{array}\right) .
$$

Note that

$$
E(x)^{-1}=\left(\begin{array}{cc}
I_{n} & 0 \\
\nabla M(x) & I_{p}
\end{array}\right),
$$

and, with 21,

$$
|E(x)| \leq 1+L_{M}, \quad\left|E(x)^{-1}\right| \leq 1+L_{M}, \quad \forall x \in \mathbb{R}^{n} .
$$

Hence, for all $v$ in $\mathbb{R}^{n+p}$ and $x \in \mathbb{R}^{n}$,

$$
\begin{aligned}
v^{\top} \mathcal{P}(\chi) v & \geq \min \{\underline{p}, \mathfrak{b}\}|E(x) v|^{2} \\
& \geq \min \{\underline{p}, \mathfrak{b}\} \frac{|v|^{2}}{\left|E(x)^{-1}\right|^{2}} \geq \frac{\min \{\underline{p}, \mathfrak{b}\}}{\left(1+L_{M}\right)^{2}}|v|^{2} .
\end{aligned}
$$

On another hand,

$$
\begin{aligned}
v^{\top} \mathcal{P}(\chi) v & \leq \max \{\bar{p}, \mathfrak{b}\}|E(x) v|^{2} \\
& \leq \max \{\bar{p}, \mathfrak{b}\}\left(1+L_{M}\right)^{2}|v|^{2} .
\end{aligned}
$$

Hence, inequality 6a holds with

$$
\overline{\mathfrak{p}}:=\max \{\bar{p}, \mathfrak{b}\}\left(1+L_{M}\right)^{2}, \quad \underline{\mathfrak{p}}:=\frac{\min \{\underline{p}, \mathfrak{b}\}}{\left(1+L_{M}\right)^{2}} .
$$

Finally, by combining inequality (30) with the definition of $\mathcal{P}$ in (31) and previous bounds, we have that inequality 6b holds with the function $\mathfrak{q}: \mathbb{R}^{n+p} \rightarrow \mathbb{R}_{>0}$ defined as

$$
\mathfrak{q}(x, \eta)=\frac{1}{\left(1+L_{M}\right)^{2}} \min \left\{\lambda_{0}, \kappa \nabla \beta(\eta-M(x))\right\},
$$

which is always positive in view of the property of $\beta$ in 23 . Finally, since the metric $\mathcal{P}$ is constant in the $z$-coordinates (indeed, by its definition, it does not depend on $z$ ), and $\Gamma_{r}$ is constant, it follows that $L_{\Gamma_{r}} \mathcal{P}(\mathcal{X})=0$ for all $\mathcal{X} \in \mathbb{R}^{n+p}$, that is $6 \mathrm{6c}$ is satisfied.

Remark 3. According to Definition 11 in Appendix, the closed-loop system is not $\delta G E S$. Indeed, the right-hand side of 33 may be not uniformly bounded from below in $(x, \eta)$ because of the properties of $\beta$ in 23 . 


\section{INCREMENTAL FORWARDING MOD $\left\{L_{g} V\right\}$}

\section{A. Design for Global (Integral) Output Regulation}

In the previous section, we have seen that under Assumptions 1 and 2, it is always possible to solve the Regional (Integral) Output Tracking Problem. Two main drawbacks of the previous procedure can be highlighted. The first concerns the fact that the proposed design relies on the solution of the PDE (10) that may be hard to compute in practice. The second is related to the fact that the condition (6d) is not satisfied as $\Gamma_{d}$ depends on $x$ and therefore only the second part of Theorem 1 can be applied (in other words, global disturbance rejection is not generically ensured).

To this end, we state a new assumption, that extends in the incremental framework the forwarding $\bmod \left\{L_{g} V\right\}$ technique presented in Section $[\mathrm{II}-\mathrm{C}$ and in particular items (i)-(iii) of Proposition 2 The proposed conditions, although more conservative, allow for a design that is easier to apply since it doesn't rely on the exact solution of the PDE (10), but only on an approximation version of it.

Assumption 3 (Incremental forwarding $\bmod \left\{L_{g} V\right\}$ ). There exist a $C^{2}$ function $\bar{M}: \mathbb{R}^{n} \mapsto \mathbb{R}^{p}$, a $C^{1}$ function $N: \mathbb{R}^{n} \mapsto$ $\mathbb{R}^{p}$ a $p \times m$ matrix $\Lambda$ and a positive real number $\lambda_{1}>0$ satisfying the following properties:

1) $L_{f_{0}} \bar{M}(x)=h(x)+N(x)$ for all $x \in \mathbb{R}^{n}$.

2) $L_{g} \bar{M}(x)=\Lambda$ for all $x \in \mathbb{R}^{n}$.

3) $\operatorname{rank}(\Lambda)=p$.

4) The following inequality holds

$$
\begin{aligned}
& L_{f_{0}} P(x)-P(x) g(x) \Psi \nabla N(x) \\
& \quad-\nabla N^{\top}(x) \Psi^{\top} g(x)^{\top} P(x) \leq-2 \lambda_{1} I,
\end{aligned}
$$

for all $x \in \mathbb{R}^{n}$, with $P$ given by Assumption 1 , and $\Psi$ defined as

$$
\Psi:=L_{g} \bar{M}(x)^{\top}\left[L_{g} \bar{M}(x) L_{g} \bar{M}(x)^{\top}\right]^{-1}=\Lambda^{\top}\left(\Lambda \Lambda^{\top}\right)^{-1} .
$$

In Assumption 3 we ask $L_{g} \bar{M}(x)$ to be constant for all $x \in \mathbb{R}^{n}$. Although such an assumption is in general much more stringent than asking for a constant rank as in Assumption 2, it is worth however noting that the definition of $\bar{M}$ is now different, see item 1 . In particular, the function $N$ represents the mismatch between the definition of $M$ in 10 and $\bar{M}$ in Assumption 3 . Such a term $N$ can be therefore used as an extra degree of freedom, to achieve the desired regularity on $L_{g} \bar{M}$ or to provide an "easy-to-compute" approximate solution of the PDE (10). In the contraction analysis, the remaining term $N$ is managed via the robustness properties of the $x$-dynamics, which is assumed to be "sufficiently contractive". This is expressed in the inequality (34). Evidently, in case $N(x)=0$ for all $x \in \mathbb{R}^{n}$, the designs of $\bar{M}$ and $M$ coincide and items 2 and 3 read as a strongest version of Assumption 2, while item 4 is automatically satisfied by Assumption 11 with $\lambda_{1}:=\lambda_{0}$. Finally, we stress that items 1-4 of Assumption 3 correspond to a generalized incremental version of the conditions (i)-(iii) of Proposition 2 when selecting $N(x)=k(x) L_{g} V(x)$.
Remark 4. Item 2 of Assumption 3 can be interpreted as the existence of a DC gain approximation $\Lambda$ of the system (15), which is constant for any linearization around any point of the state space.

Based on the previous assumption, we focus on a control law of the form

$$
\phi(x, \eta)=\phi_{0}(x)+\kappa \Psi(\eta-\bar{M}(x))-\Psi N(x),
$$

where the matrix $\Psi$ is defined as in (35), $N$ is the function of item 1 satisfying (34), and $\kappa \in \mathbb{R}$ is a control gain parameter. We have then the following result.

Proposition 4 (Incremental uniform forwarding $\bmod \left\{L_{g} V\right\}$ ). Consider system (1) and suppose Assumptions 1 and 3 hold. Suppose, moreover, that there exists a positive real number $L_{M}>0$ such that

$$
\|\nabla \bar{M}(x)\| \leq L_{M}
$$

holds for all $x \in \mathbb{R}^{n}$. Then, for any $\kappa>0$, the closedloop system (17)-(2) with $\phi$ selected as in (36), satisfies the properties (6a), (6b), (6c), (6d) and the conditions of item 1 of Theorem 17 namely, there exists $\mathfrak{q}>0$ such that the function $\mathfrak{q}$ in 6b satisfies $\mathfrak{q}(\mathcal{X}) \geq \underline{\mathfrak{q}}$ for all $\mathcal{X} \in \mathbb{R}^{n}$.

A direct consequence of Proposition 3 and Theorem 1 is the following result. The proof is omitted for compactness.

Corollary 2. Under the assumptions of Proposition 4 the control law (2b), with $\phi$ selected as in [36, satisfies the Global (Integral) Output Regulation Problem for system (1).

Proof of Proposition 4 . We follow here the main steps of the proof of Proposition 3 . Consider the change of coordinates (24). By item 2 of Assumption 3 and by definition of $\Psi$ in (35), we have $L_{g} \bar{M}(x) \Psi=I_{p}$. Hence, the $z$-dynamics reads

$$
\begin{aligned}
\dot{z} & =h(x)-L_{f} \bar{M}(x)-L_{g} \bar{M}(x) \phi(x, \eta) \\
& =h(x)-L_{f_{0}} \bar{M}(x)-\kappa L_{g} \bar{M}(x) \Psi z+L_{g} \bar{M}(x) \Psi N(x) \\
& =-\kappa z,
\end{aligned}
$$

where we used the relation on $h, \bar{M}, N$ given in item 1) of Assumption 3 The closed-loop system (1), 2), 36) can be then compactly written as

$$
\dot{\chi}=F(\chi)+\widetilde{\Gamma}(\mathcal{X}) w
$$

with $F$ and $\widetilde{\Gamma}$ defined as

$$
\begin{aligned}
& F(\chi):=\left(\begin{array}{c}
f_{0}(x)+\kappa g(x) \Psi z-g(x) \Psi N(x) \\
-\kappa z
\end{array}\right), \\
& \widetilde{\Gamma}(\chi):=\left(\begin{array}{cc}
g(x) & 0 \\
-\Lambda & -I_{p}
\end{array}\right) .
\end{aligned}
$$

The Jacobian $J_{F}$ of $F$ is of the form (25) where now the components $J_{11}, J_{12}$ and $J_{22}$ are defined as

$$
\begin{aligned}
& J_{11}(\chi):=\nabla f_{0}(x)+\nabla g(x) \Psi(\kappa-N(x))-g(x) \Psi \nabla N(x), \\
& J_{12}(\chi):=\kappa g(x) \Psi, \quad J_{22}(\chi):=-\kappa I_{p} .
\end{aligned}
$$


Consider the metric $\overline{\mathcal{P}}$ defined in $(26)$ for some $\mathfrak{b}>0$ to be defined later, and the matrix function $\mathcal{L}: \mathbb{R}^{n+p} \rightarrow$ $\mathbb{R}^{(n+p) \times(n+p)}$ defined as

$$
\mathcal{L}(\chi):=L_{F} \overline{\mathcal{P}}(\chi)+\left(\begin{array}{cc}
\lambda_{1} I_{n} & 0 \\
0 & \kappa I_{p}
\end{array}\right)
$$

with $\lambda_{1}$ given by (34). By construction, $\mathcal{L}$ takes symmetric values. We want to show that for any value of $\kappa$, the function $\mathcal{L}$ so defined is negative definite for all $\chi \in \mathbb{R}^{n+p}$. By inspecting its components as in 28), and by using the Killing vector property $L_{g} P(x)=0$ in (17), we obtain

$$
\begin{aligned}
\mathcal{L}_{11}(\chi):= & L_{f_{0}} P(x)+\lambda_{1} I_{n}-P(x) g(x) \Psi \nabla N(x) \\
& -\nabla N^{\top}(x) \Psi^{\top} g^{\top}(x) P(x), \\
\mathcal{L}_{12}(\chi):= & \kappa P(x) g(x) \Psi, \quad \mathcal{L}_{22}(\chi):=-\kappa(2 \mathfrak{b}-1) I_{p} .
\end{aligned}
$$

Trivially, $\mathcal{L}_{22}(\chi)<0$ for all $\chi \in \mathbb{R}^{n+p}$ and for any $\mathfrak{b}>\frac{1}{2}$. As a consequence, $\mathcal{L}(\chi)$ is negative definite for all $\chi \in \mathbb{R}^{n+p}$ if its Schur complement, denoted as $\mathrm{SC}_{\mathcal{L}}(\chi)$, is also negative definite for all $\chi \in \mathbb{R}^{n+p}$. By using [34], we compute

$$
\begin{aligned}
\mathrm{SC}_{\mathcal{L}}(\chi) & :=\mathcal{L}_{11}(\chi)-\mathcal{L}_{12}(\chi) \mathcal{L}_{22}(\chi)^{-1} \mathcal{L}_{12}(\chi)^{\top} \\
& \leq-\lambda_{1} I_{n}+\frac{\kappa}{2 \mathfrak{b}-1} P(x) g(x) \Psi \Psi^{\top} P(x) g(x) P(x) \\
& \leq-\left(\lambda_{1}-\frac{\kappa\left(\bar{p} \bar{g} k_{1}\right)^{2}}{2 \mathfrak{b}-1}\right) I_{n},
\end{aligned}
$$

where $k_{1}=\sqrt{\left\|\Psi \Psi^{\top}\right\|}$. Hence, by selecting

$$
\mathfrak{b}=\frac{1}{2}\left(1+\frac{\kappa\left(\bar{p} \bar{g} k_{1}\right)^{2}}{\lambda_{1}}\right) .
$$

we obtain $\mathrm{SC}_{\mathcal{L}}(x) \leq 0$ and consequently $\mathcal{L}(\chi) \leq 0$ for all $\chi \in \mathbb{R}^{n}$. Consequently, recalling the definition of $\mathcal{L}$ in 38, it yields

$$
L_{F} \overline{\mathcal{P}}(\chi) \leq-\min \left\{\lambda_{1}, \kappa\right\} I_{n+p} .
$$

Moreover, in light of the structure of $\widetilde{\Gamma}$ and the fact that $\overline{\mathcal{P}}$ is independent of $z$, we deduce that $L_{\widetilde{\Gamma}} \overline{\mathcal{P}}(\chi)=0$ for all $\chi \in \mathbb{R}^{n+p}$. Finally, as in the proof of Proposition 3 consider the function $\mathcal{P}$ defined as

$$
\mathcal{P}(\chi):=\bar{E}(x)^{\top} \overline{\mathcal{P}}(\mathcal{X}) \bar{E}(x), \bar{E}(x):=\left(\begin{array}{cc}
I_{n} & 0 \\
-\nabla \bar{M}(x) & I_{p}
\end{array}\right),
$$

to obtain a metric in the original coordinates $\mathcal{X}=(x, \eta)$. The rest of the proof follows the same lines of the proof of Proposition 3, with $\overline{\mathfrak{p}}, \mathfrak{p}$ selected as in (32),

$$
\underline{\mathfrak{q}}:=\frac{1}{\left(1+L_{M}\right)^{2}} \min \left\{\lambda_{1}, \kappa\right\},
$$

and by recalling that the Killing vector property is invariant with respect to global diffeomorphism (see Remark 7 in Appendix).

Remark 5. The fact that the closed-loop defines a uniform global contraction with respect to a metric $\mathcal{P}(\chi)$ and that $\Gamma$ is of Killing with respect to such $\mathcal{P}$, is a sufficient condition to show that the closed loop is Incremental ISS ([4]) with respect to the input $w$. Therefore, in case $w=(d, r)$ is a time-varying bounded and integrable signal, the trajectory of the closed loop is guaranteed to be bounded for all positive times. Moreover, if $\lim _{t \mapsto \infty} w(t)=w^{\star}$ for some constant $w^{\star}$, then the closed loop trajectory asymptotically tends to an equilibrium point where constant output regulation is achieved.

\section{B. (Partial) Output Feedback Design}

In this section we explore the case in which the control law used to stabilize the cascade system is not dependent on $x$ but only on the state of the integrator itself (see, e.g., [10] or, more recently, [39]). Solutions based on forwarding in which the stabilization of the cascade system is achieved by a control law only dependent on the local state are not new in literature. For linear systems, this corresponds to the use of a pure $I$-regulator with a small gain, instead of a classical PI-regulator. In this section we show how, by slightly strengthening the condition (34), the feedback law (36) can be modified into

$$
\phi(x, \eta)=\phi_{0}(x)+\kappa \Psi \eta
$$

where $\phi_{0}$ is the pre-stabilizing feedback needed to achieve contractivity of the $x$-dynamics (recall that for an open-loop contractive system, $\phi_{0}$ can be zero), and $\kappa>0$ is a small positive gain to be selected. With respect to control law (36), the term $\kappa \Psi \bar{M}(x)+N(x)$ is not needed. To this end, we state the following assumption (which is new with respect to [39]).

Assumption 4 (Generalized forwarding for output feedback). There exist positive real numbers $\mathfrak{a}, \lambda_{2}>0$ such that all the items of Assumption 3 are verified and moreover the following inequality

$$
\begin{array}{r}
\left(\mathfrak{a} P(x) g(x) \Psi-\frac{1}{\mathfrak{a}} \nabla N^{\top}(x)\right)\left(\mathfrak{a} P(x) g(x) \Psi-\frac{1}{\mathfrak{a}} \nabla N^{\top}(x)\right)^{\top} \\
+L_{f_{0}} P(x) \leq-2 \lambda_{2} I_{n}
\end{array}
$$

holds for all $x \in \mathbb{R}^{n}$.

Remark 6. Simple computation show that (42) implies (34) for some $\lambda_{1} \geq \lambda_{2}$. Moreover, if $N(x)=0$ for all $x \in \mathbb{R}^{n}$, the inequality (42) is always verified under Assumption 1$]$ In particular, it can be directly derived from inequality (16) by selecting

$$
\mathfrak{a}=\frac{1}{\bar{p} \bar{g}} \sqrt{\frac{\lambda_{0}}{2\left\|\Psi \Psi^{\top}\right\|}}, \quad \lambda_{2}=\frac{\lambda_{0}}{2},
$$

and $q, \bar{p}, \bar{g}$ as in (16), (17), and 21).

Proposition 5 (Incremental uniform integral action). Consider system (1) and suppose Assumptions 1 and 4 hold and that there exists $L_{M} \in \mathbb{R}_{>0}$ such that the inequality (37) holds for all $x \in \mathbb{R}^{n}$. Then, there exists a positive real number $\bar{\kappa}>0$, such that, for any $\kappa \in(0, \bar{\kappa}]$, the closed-loop system (1)-(2), with $\phi$ selected as in (41), satisfies the properties (6a), (6b), (6c), 6d) and the conditions of item 1 of Theorem 1 . namely, there exists $\mathfrak{q}>0$ such that the function $\mathfrak{q}$ in $6 \mathrm{~b}$ satisfies $\mathfrak{q}(\mathcal{X}) \geq \underline{\mathfrak{q}}$ for all $\mathcal{X} \in \mathbb{R}^{n}$. 
Combining Proposition 5 with Theorem 1, we directly obtain the following result.

Corollary 3. Under the assumptions of Proposition 5 the control law (2b), with $\phi$ selected as in (41), satisfies the Global (Integral) Output Regulation Problem for system (1) for $\kappa>0$ small enough.

Proof of Proposition 5. We follow the same steps used in the proof of Proposition 4 . By using the change of coordinates (24), and defining $\chi=(x, z)$, the closed-loop system (1), (2), (41) reads as

$$
\dot{\chi}=F(\chi)+\widetilde{\Gamma}(\mathcal{X}) w
$$

with $F$ and $\widetilde{\Gamma}$ defined as

$$
\begin{aligned}
& F(\chi):=\left(\begin{array}{c}
f_{0}(x)+\kappa g(x) \Psi(z+\bar{M}(x)) \\
-\kappa z-N(x)-\kappa \bar{M}(x)
\end{array}\right), \\
& \widetilde{\Gamma}(\chi):=\left(\begin{array}{cc}
g(x) & 0 \\
-\Lambda & -I_{p}
\end{array}\right) .
\end{aligned}
$$

The Jacobian $J_{F}$ of $F$ is now of the form

$$
J_{F}(\chi):=\nabla F \chi(\chi)=\left(\begin{array}{ll}
J_{11}(\chi) & J_{12}(\chi) \\
J_{21}(\chi) & J_{22}(\chi)
\end{array}\right),
$$

where now the components $J_{11}, J_{12}, J_{22}$ and $J_{22}$ are defined as

$$
\begin{aligned}
J_{11}(\chi):= & \nabla f_{0}(x)+\kappa \nabla g(x) \Psi(z+\bar{M}(x)) \\
& +\kappa g(x) \Psi \nabla \bar{M}(x), \\
J_{12}(\chi):= & \kappa g(x) \Psi, \quad J_{21}(\chi):=-\kappa \nabla \bar{M}(x)-\nabla N(x), \\
J_{22}(\chi):= & -\kappa I_{p} .
\end{aligned}
$$

Consider now the metric $\overline{\mathcal{P}}$ defined in $(26)$ for some $\mathfrak{b}>0$ to be defined later, and the function $\mathcal{L}: \mathbb{R}^{n+p} \rightarrow \mathbb{R}^{(n+p) \times(n+p)}$ defined as

$$
\mathcal{L}(\chi):=L_{F} \overline{\mathcal{P}}(\chi)+\left(\begin{array}{cc}
\lambda_{2} I_{n} & 0 \\
0 & \frac{1}{2} \kappa \mathfrak{b} I_{p}
\end{array}\right)
$$

with $\lambda_{2}$ given by (42). By construction, $\mathcal{L}$ takes symmetric values. We want to show that for any value of $\kappa$, the function $\mathcal{L}$ so defined is negative definite for all $\chi \in \mathbb{R}^{n+p}$. By inspecting its components as in (28), and by using the Killing vector property $L_{g} P(x)=0$ in (17), we obtain

$$
\begin{aligned}
\mathcal{L}_{11}(\chi):= & L_{f_{0}} P(x)+\lambda_{2} I_{n}+\kappa P(x) g(x) \Psi \nabla \bar{M}(x) \\
& +\kappa \nabla \bar{M}^{\top}(x) \Psi^{\top} g^{\top}(x) P(x), \\
\mathcal{L}_{12}(\chi):= & \kappa P(x) g(x) \Psi-\kappa \mathfrak{b} \nabla \bar{M}^{\top}(x)-\mathfrak{b} \nabla N^{\top}(x), \\
\mathcal{L}_{22}(\chi):= & -\frac{3}{2} \kappa \mathfrak{b} I_{p} .
\end{aligned}
$$

Trivially, $\mathcal{L}_{22}(\chi)<0$ for all $\chi \in \mathbb{R}^{n+p}$ and for any $\mathfrak{b}>0$. As a consequence, $\mathcal{L}(\chi)$ is negative definite for all $\chi \in \mathbb{R}^{n+p}$ if its Schur complement, denoted as $\mathrm{SC}_{\mathcal{L}}(\chi)$, is also negative definite for all $\chi \in \mathbb{R}^{n+p}$. By using the inequality

$$
(a+b)(a+b)^{\top} \leq(1+\nu) a a^{\top}+\left(1+\nu^{-1}\right) b b^{\top}
$$

valid for any matrices $a, b$ of suitable dimension and any $\nu>0$, we obtain

$$
\begin{array}{r}
\operatorname{SC}_{\mathcal{L}}(\chi)=-\mathcal{L}_{12}(\chi) \mathcal{L}_{22}(\chi)^{-1} \mathcal{L}_{12}(\chi)^{\top} \\
=\frac{2}{3 \kappa \mathfrak{b}}\left[\kappa P(x) g(x) \Psi-\kappa \mathfrak{b} \nabla \bar{M}^{\top}(x)-\mathfrak{b} \nabla N^{\top}(x)\right] \times \\
\times\left[\kappa P(x) g(x) \Psi-\kappa \mathfrak{b} \nabla \bar{M}^{\top}(x)-\mathfrak{b} \nabla N^{\top}(x)\right]^{\top} \\
\leq\left(\sqrt{\frac{\kappa}{\mathfrak{b}}} P(x) g(x) \Psi-\sqrt{\frac{\mathfrak{b}}{\kappa}} \nabla N^{\top}(x)\right) \times \\
\times\left(\sqrt{\frac{\kappa}{\mathfrak{b}}} P(x) g(x) \Psi-\sqrt{\frac{\mathfrak{b}}{\kappa}} \nabla N^{\top}(x)\right)^{\top} \\
+2 \kappa \mathfrak{b} \nabla \bar{M}^{\top}(x) \nabla \bar{M}(x) .
\end{array}
$$

Hence, select $\mathfrak{b}=\kappa / \mathfrak{a}^{2}$ with $\mathfrak{a}$ given by $(42$ ). It gives

$$
\begin{aligned}
& \operatorname{SC}_{\mathcal{L}}(\chi) \leq 2 \kappa \mathfrak{b} \nabla \bar{M}^{\top}(x) \nabla \bar{M}(x) \\
& +\left(\mathfrak{a} P g(x) \Psi-\frac{1}{\mathfrak{a}} \nabla N^{\top}(x)\right)\left(\mathfrak{a} P g(x) \Psi-\frac{1}{\mathfrak{a}} \nabla N^{\top}(x)\right)^{\top}
\end{aligned}
$$

and therefore, by using (42), and the bounds (16), 177) and (21), we obtain

$$
\mathrm{SC}_{\mathcal{L}}(\chi) \leq-\left(\lambda_{2}-2 \kappa \bar{p} \bar{g}\|\Psi\| L_{M}-2 \kappa^{2} \frac{L_{M}^{2}}{\mathfrak{a}^{2}}\right) I_{n},
$$

for all $\chi \in \mathbb{R}^{n+p}$. Hence by selecting $\bar{\kappa}>0$ small enough so that

$$
\lambda_{2}-2 \bar{\kappa} \bar{p} \bar{g}\|\Psi\| L_{M}-2 \bar{\kappa}^{2} \frac{L_{M}^{2}}{\mathfrak{a}^{2}} \geq 0
$$

and by recalling the definition of $\mathcal{L}$ in $(43)$, and the structure of $\widetilde{\Gamma}$, we conclude that

$$
L_{F} \overline{\mathcal{P}}(\chi) \leq-\min \left\{\lambda_{2}, \frac{\kappa^{2}}{2 \mathfrak{a}^{2}}\right\}, \quad L_{\widetilde{\Gamma}} \mathcal{P}(\chi)=0
$$

for all $\chi \in \mathbb{R}^{n}$ and for all $\kappa \in(0, \bar{\kappa}]$. The rest of the proof follows the same lines of the proof of Proposition 4 with $\underline{\mathfrak{q}}:=\frac{1}{\left(1+L_{M}\right)^{2}} \min \left\{\lambda_{2}, \frac{\kappa^{2}}{2 \mathfrak{a}^{2}}\right\}$.

\section{Academic Example}

Consider a nonlinear system of the form (1) with $n=3$, $m=3, p=2$ and functions $f, g, h$ selected as

$$
\begin{aligned}
& f(x)=\left(\begin{array}{c}
-x_{1}-x_{1}^{3} \\
-x_{2}-\frac{1}{2} x_{2} \cos \left(x_{2}\right) \\
-2 x_{3}
\end{array}\right), g(x)=\left(\begin{array}{cc}
1 & 1 \\
2+\cos \left(x_{2}\right) & 0 \\
0 & 1
\end{array}\right) \\
& h(x)=\left(\begin{array}{c}
x_{1}^{3}-2 x_{2}-\sin \left(x_{2}\right) \\
x_{3}
\end{array}\right)
\end{aligned}
$$

Following [17, Chapter 5], we compute

$$
L_{g} h(x)=\left(\begin{array}{cc}
3 x_{1}^{2}-\left(2+\cos \left(x_{2}\right)\right)^{2} & 3 x_{1}^{2} \\
0 & 1
\end{array}\right)
$$

which is not invertible for all $x$, as it contains some singularities, for instance in $x_{1}= \pm \sqrt{3}$ and $x_{2}=1$. Therefore, it does not exist a global diffeomorphism transforming this system into a normal form [17, Chapter 5]. Nevertheless, it's possible to check that Assumptions 1 and 3 hold. First, we can check that inequality (16) is verified with $\phi_{0}(x)=0$, $P(x)=\operatorname{diag}\left(1,\left(2+\cos \left(x_{2}\right)\right)^{2}, \frac{1}{2}\right)$ and $\lambda_{0}=1$. Indeed,

$$
L_{f} P(x)=\operatorname{diag}\left(-2-6 x_{1}^{2},-1,-2\right) \leq-I .
$$


Furthermore, it can be verified that the Killing vector property (17) is verified. Hence Assumption 1 holds. Then, selecting

$$
\begin{array}{ll}
\bar{M}(x)=\left(\begin{array}{c}
-x_{1} \\
-\frac{1}{2} x_{3}
\end{array}\right), & \Lambda=\left(\begin{array}{cc}
-1 & -1 \\
0 & -\frac{1}{2}
\end{array}\right), \\
N(x)=\left(\begin{array}{cc}
x_{1}+2 x_{2}+\sin \left(x_{2}\right) \\
0
\end{array}\right), & \Psi=\left(\begin{array}{cc}
-1 & 2 \\
0 & -2
\end{array}\right) .
\end{array}
$$

verifies items 1-3 of Assumption 3 . Finally, item 4 and in particular inequality (34) is satisfied for $\lambda_{1}=\frac{1}{2}$. Hence, Corollary 2 holds and the control law (36), reading

$$
\begin{aligned}
\dot{\eta} & =h(x)-r=\left(\begin{array}{c}
x_{1}^{3}-2 x_{2}-\sin \left(x_{2}\right)-r_{1} \\
x_{3}-r_{2}
\end{array}\right) \\
u & =\phi(x, \eta) \\
& =\left(\begin{array}{c}
-\kappa \eta_{1}+2 \kappa \eta_{2}+(1-\kappa) x_{1}+(2+\kappa) x_{2}+\sin \left(x_{2}\right) \\
-\kappa\left(\eta_{2}+x_{3}\right)
\end{array}\right),
\end{aligned}
$$

solves the Global (Integral) Output Regulation Problem for any $\kappa>0$.

\section{CASE STUdies}

\section{A. Lipschitz systems}

In this section we specialize our previous results to the following class of Lipschitz systems of the form

$$
\begin{aligned}
\dot{x} & =A x+B(u+d)+S \vartheta(H x) \\
e & =C x+D \vartheta(H x)-r
\end{aligned}
$$

where $A, B, C, S, D, H$ are constant matrices of suitable dimension and $\vartheta$ is a $C^{1}$ Lipschitz function with Lipschitz constant $\vartheta_{L}$. We suppose that the following assumption holds. Assumption 11. There exist a constant symmetric positive definite matrix $P$ and a positive real number $\lambda_{0}>0$ satisfying the inequality

$$
P A+A^{\top} P+P S \frac{\partial \vartheta}{\partial x}(x) H+H^{\top} \frac{\partial \vartheta^{\top}}{\partial x}(x) S^{\top} P \leq-2 \lambda_{0} I
$$

for all $x \in \mathbb{R}^{n}$.

Such an assumption is a particular case of Assumption 1 . in which we considered a constant Euclidean metric $P$. Assumption 11 can be satisfied after a preliminary statefeedback, by following for instance [3] (see also references therein). Following the ideas of Section IV instead of looking for the exact solution $M$ of the PDE (10), we look for an approximation $\bar{M}$ that is obtained by considering only the linear terms of (44). Let us define the following functions and matrices

$$
\begin{gathered}
\bar{M}(x)=C A^{-1} x, \quad \Lambda:=C A^{-1} B \\
N(x):=\left(C A^{-1} D-S\right) \vartheta(H x), \quad \Psi=\Lambda^{\top}\left(\Lambda \Lambda^{\top}\right)^{-1} .
\end{gathered}
$$

With these definitions, it follows that, by using the Lipschitz constant of $\vartheta$, inequality (34) in Assumption 3 is satisfied if

$$
\begin{array}{r}
P B \Psi\left(C A^{-1} D-S\right) H+\left[P B \Psi\left(C A^{-1} D-S\right) H\right]^{\top} \\
\leq \frac{2\left(\lambda_{0}-\lambda_{1}\right)}{\vartheta_{L}} I
\end{array}
$$

for some $\lambda_{1}>0$. Note that inequality [45] is verified with $\lambda_{1}=\lambda_{0}$ when $C A^{-1} D=S$ or for some $\lambda_{1}<\lambda_{0}$ when $\vartheta_{L}$ is sufficiently small compared to $\lambda_{0}$. Furthermore, inequality (42) in Assumption 4 reads

$$
\begin{aligned}
(\mathfrak{a} P B \Psi- & \left.\frac{\vartheta_{L}}{\mathfrak{a}}\left(C A^{-1} D-S\right) H\right) \\
\left(\mathfrak{a} P B \Psi-\frac{\vartheta_{L}}{\mathfrak{a}}\left(C A^{-1} D-S\right) H\right)^{\top} & \\
& \leq 2\left(\lambda_{0}-\lambda_{2}\right) I
\end{aligned}
$$

for some $\lambda_{2}, \mathfrak{a}>0$. Hence, the following can be stated.

Corollary 4. Consider system (44) and suppose Assumption 17' holds. Then, the following holds.

- If $\Lambda$ is full rank and the inequality (45) is satisfied for some $\lambda_{1}>0$, the regulator

$$
\begin{aligned}
& \dot{\eta}=e, \\
& u=\kappa \Psi\left(\eta-C A^{-1} x\right)-\Psi\left(C A^{-1} D-S\right) \vartheta(H x)
\end{aligned}
$$

solves the (Global) Integral Output Regulation Problem for any $\kappa>0$.

- Moreover if the inequality (46) is satisfied for some $\lambda_{2}, \mathfrak{a}>0$, then the output-feedback control law

$$
\begin{aligned}
& \dot{\eta}=e \\
& u=\kappa \Psi \eta
\end{aligned}
$$

solves the (Global) Integral Output Regulation Problem for $\kappa$ sufficiently small.

\section{B. Systems in Normal Form}

Most of the results in output regulation literature focus on systems possessing a well defined relative degree ([17, Chapter IV]). See, for instance, [6], [19], [21]. In the case of unitary relative degree (the extension to higher relative degree can dealt with canonical tools, see, e.g., [36, Section V]), system (1) can be rewritten as

$$
\begin{aligned}
& \dot{z}=\psi(z, y) \\
& \dot{y}=q(z, y)+b(z, y)(u+d), \\
& e=y-r
\end{aligned}
$$

where $z \in \mathbb{R}^{n-1}$ is the so-called zero-dynamics, $y \in \mathbb{R}$ is the output to be regulated, $u \in \mathbb{R}$ is the control, $r \in \mathbb{R}$ is the reference and $d \in \mathbb{R}$ represents some constant perturbation.

By using the change of coordinates $y \mapsto e:=y-r$, system 477 can be alternatively rewritten as

$$
\dot{z}=\bar{\psi}(w, z, e), \quad \dot{e}=\bar{q}(w, z, e)+\bar{b}(w, z, e) u,
$$

with $\bar{\psi}(w, z, e):=\psi(z, e+r), \bar{q}(w, z, e):=q(z, e+r)+$ $b(z, e+r) d, \bar{b}(w, z, e):=b(z, e+r)$. Concerning the zerodynamics, it is worth recalling that very few work addressed the case of non-minimum-phase, that is, when the dynamics

$$
\dot{z}=\bar{\psi}(w, z, 0)
$$

is possibly unstable. Most of the works, indeed, focused on the case in which such a zero-dynamics possess a unique steady-state trajectory (possibly that depends on $w$ ) which is attractive with a given domain of attraction. See, for instance, [36. Assumption V.1], [21. Assumption 4], [24, Assumption 
4], [37, Assumption 4], [26, Assumption H2], and others. This, in turn, corresponds to ask that the subsystem (48) possesses some incremental stability property on a given set of interest, since the attractivity properties are uniform with respect to $w$. In the global framework considered in this work, this corresponds to our Assumption 1.

We focus here on minimum-phase systems (47) possessing a constant, and therefore, without loss of generality, unitary high frequency gain, that is $b(z, y)=1$. System (47) reads

$$
\begin{aligned}
& \dot{z}=\psi(z, y) \\
& \dot{y}=q(z, y)+u+d \\
& e=y-r .
\end{aligned}
$$

In our framework, the minimum-phase assumption is stated as follows.

Assumption 5 (Minimum Phase). Consider system (49). There exist positive real numbers $\bar{q}, \bar{\psi}_{y}, \bar{p}_{z}, \underline{p}_{z}, \lambda_{z}>0$ and a function $P_{z}: \mathbb{R}^{n-1} \mapsto \mathbb{R}^{(n-1) \times(n-1)}$ taking symmetric positive values so that the following inequalities ${ }^{2}$ hold

$$
\begin{aligned}
\|\nabla q(z, y)\| \leq \bar{q}, & \left\|\nabla_{y} \psi(z, y)\right\| \leq \bar{\psi}_{y}, \quad \text { (50) } \\
\underline{p}_{z} I_{n-1} \leq P_{z}(z) \leq \bar{p}_{z} I_{n-1}, & L_{\psi} P_{z}(z, y) \leq-2 \lambda_{z} I_{n}
\end{aligned}
$$

for all $(z, y) \in \mathbb{R}^{n}$.

We have the following result.

Proposition 6 (Output regulation for minimum phase). Suppose Assumption 5 is satisfied. Then, there exists a positive real number $\underline{\sigma} \geq 1$ such that, for any fixed $\sigma \geq \underline{\sigma}$, Assumptions 1,3 and 4 are satisfied with

$$
\begin{gathered}
P(z, y)=\left(\begin{array}{cc}
P_{z}(z) & 0 \\
0 & \sigma^{-\frac{2}{3}}
\end{array}\right), \quad \underline{p}=\min \left\{\underline{p}_{z}, \sigma^{-\frac{2}{3}}\right\}, \\
\left.\phi_{0}(z, y)=-\sigma y, \quad \lambda_{0}=\frac{\lambda_{z}}{2}, \quad \lambda_{1}=\lambda_{2}=\frac{\lambda_{z}}{4}, 1\right\}, \quad \mathfrak{a}=\sigma^{-\frac{2}{3}}, \\
\bar{M}(z, y)=-\frac{y}{\sigma}, \quad N(z, y)=-\frac{q(z, y)}{\sigma}, \Lambda=\Psi^{-1}=-\frac{1}{\sigma},
\end{gathered}
$$

with $P_{z}, \bar{p}_{z}, \underline{p}_{z}$ and $\lambda_{z}$ given by Assumption 5 .

As a consequence of previous proposition, we have the following corollary that can be derived from Proposition 6 and Corollary 3 .

Corollary 5. Under the assumptions of Proposition 6 the control law

$$
\dot{\eta}=e, \quad u=-\sigma(y+\kappa \eta)
$$

solves the Global (Integral) Output Regulation Problem for system 49f for $\kappa>0$ small enough and $\sigma>0$ large enough.

Proof of Proposition 6 Let $\underline{\sigma}$ be defined as

$$
\begin{array}{r}
\underline{\sigma}^{\frac{1}{3}}:=\max \left\{\bar{q}+\frac{\lambda_{z}}{2}+\frac{\left(\bar{p}_{z} \bar{\psi}_{y}+\bar{q}\right)^{2}}{2 \lambda_{z}}, 2 \sqrt{\frac{\bar{q}}{\lambda_{z}}},\right. \\
\left.\sqrt{\frac{2}{\lambda_{z}}}(1+2 \bar{q}), 1\right\} .
\end{array}
$$

\footnotetext{
${ }^{2}$ The notation $L_{\psi} P_{z}(z, y)$ has to be understood as the Lie derivative of $P_{z}$ along the vector field $z \mapsto \psi(z, y)$ where $y$ is fixed.
}

Now, with the control $u=\psi_{0}(x, z)$ system (49) is in the form (1) and (15) with $x=(z, y), f_{0}(x)=\left(\psi^{\top}(z, y), q(z, y)-\right.$ $\sigma y)^{\top}, g(x)=(0,1)^{\top}, h(x)=y$. With the function $P$ defined as in the statement of the proposition, let us define

$$
\mathcal{R}(x):=L_{f_{0}} P(x)+\lambda_{z} I_{n}=\left(\begin{array}{cc}
\mathcal{R}_{11}(x) & \mathcal{R}_{12}(x) \\
\mathcal{R}_{12}(x)^{\top} & \mathcal{R}_{22}(x)
\end{array}\right),
$$

where

$$
\begin{aligned}
& \mathcal{R}_{11}(x):=L_{\psi} P_{z}(z)+\lambda_{z} I_{n} \\
& \mathcal{R}_{12}(x):=P_{z}(z) \nabla_{y} \psi(z, y)+\sigma^{-\frac{2}{3}} \nabla_{z} q(z, y), \\
& \mathcal{R}_{22}(x):=-2 \sigma^{\frac{1}{3}}+2 \sigma^{-\frac{2}{3}} \nabla_{y} q(z, y)+\lambda_{z} .
\end{aligned}
$$

By using (51) and the definition of $\underline{\sigma}$ in (52), we have $\mathcal{R}_{11}(x) \leq 0$ and $\mathcal{R}_{22}(x) \leq 0$ for all $x \in \mathbb{R}^{n}$ and all $\sigma \geq \underline{\sigma}$. Then, by denoting with $\mathrm{SC}_{\mathcal{R}}$ the Schur complement of $\mathcal{R}$, and by using (50) and $\sigma^{-\frac{2}{3}} \leq 1$, we compute

$$
\mathrm{SC}_{\mathcal{R}}(x) \leq-\left(\lambda_{z}-\frac{\left(\bar{p}_{z} \bar{\psi}_{z}+\bar{q}\right)^{2}}{2 \sigma^{\frac{1}{3}}-2 \bar{q}-\lambda_{z}}\right) I_{n} \leq 0 \quad \forall x \in \mathbb{R}^{n},
$$

for all $\sigma \geq \underline{\sigma}$. Therefore $\mathcal{R}(x) \leq 0$ for all $x \in \mathbb{R}^{n}$ which shows inequality (16) with $\lambda_{0}$ selected as in the statement of the theorem. Finally, since the function $P$ defined in the statement of the proposition and the function $g$ are constant in the $y$-coordinates (and zero otherwise), the Killing vector property $L_{g} P(x)=0$ is trivially satisfied. This shows that Assumption 1 holds. Note that an alternative proof can be done, in the case of constant metric $P_{z}$, by following [30. Theorem 2].

Now, in order to show Assumption 3 first note that with the definition of $\bar{M}, N, \Lambda, \Psi$ given in the statement, item 1, 2 and 3 are trivially satisfied with simple computations. Then, to show inequality (34), we define

$$
Q_{1}(x):=-P(x) g(x) \Psi \nabla N(x)-\nabla N^{\top}(x) \Psi^{\top} g(x)^{\top} P(x) .
$$

By omitting computations and using inequality $(50)$, we have

$$
Q_{1}(x)=\sigma^{-\frac{2}{3}}\left(\begin{array}{cc}
0 & \nabla_{z} q(z, y) \\
\nabla_{z} q^{\top}(z, y) & \nabla_{y} q(z, y)
\end{array}\right) \leq 2 \sigma^{-\frac{2}{3}} \bar{q} I_{n},
$$

that is, $Q_{1}(x) \leq \frac{\lambda_{z}}{2} I_{n}$ for all $x \in \mathbb{R}^{n}$ and all $\sigma \geq \underline{\sigma}$. Therefore, by recalling the definition of $\mathcal{R}$ given in (53) and recalling that $\mathcal{R}(x) \leq 0$ for all $x \in \mathbb{R}^{n}$, inequality (34) reads $L_{f_{0}} P(x)+Q_{1}(x) \leq \mathcal{R}(x)-\frac{\lambda_{z}}{2} I_{n} \leq-\frac{\lambda_{z}}{2} I_{n}$ for all $x \in \mathbb{R}^{n}$ and for all $\sigma \geq \underline{\sigma}$. Hence, inequality (34) holds with $\lambda_{1}$ given as in the statement of the proposition showing Assumption 3

Finally, in order to show Assumption 4 , we define

$Q_{2}(x):=T(x) T^{\top}(x), T(x):=\mathfrak{a} P(x) g(x) \Psi-\frac{1}{\mathfrak{a}} \nabla N^{\top}(x)$, which gives $T^{\top}(x)=\sigma^{-\frac{1}{3}}\left(\nabla_{z} q(z, y), \nabla_{y} q(z, y)-1\right)$ with $\mathfrak{a}$ selected as in the statement of the Proposition. By using the bounds in $(50)$, we obtain $Q_{2}(x) \leq\|T(x)\|^{2} I_{n} \leq \sigma^{-\frac{2}{3}}(2 \bar{q}+$ $1)^{2} I_{n} \leq \frac{\lambda z}{2} I_{n}$ for all $x \in \mathbb{R}^{n}$ and all $\sigma \geq \underline{\sigma}$. Hence, by following previous steps, we obtain $L_{f_{0}} P(x)+Q_{2}(x) \leq$ $\mathcal{R}(x)-\frac{\lambda_{z}}{2} I_{n} \leq-\frac{\lambda_{z}}{2} I_{n}$ for all $x \in \mathbb{R}^{n}$ and for all $\sigma \geq \underline{\sigma}$. This shows inequality (42) with $\lambda_{2}$ given as in the statement of the proposition and concludes the proof. 


\section{Conclusions}

We studied the problem of designing an integral-action based state-feedback control law for multi-input multi-output input-affine nonlinear systems following contraction arguments. We developed an extension of the forwarding via exact change of coordinates [27] and forwarding $\bmod \left\{L_{g} V\right\}$ [33] approaches in an incremental framework. The analysis is done via a metric approach rather than using an incremental Lyapunov approach. The resulting control law is applied in global output regulation problems for nonlinear systems in which both perturbations and references are supposed to be constant and arbitrarily large. The proposed approach is coordinate-free since no use of normal form is required. The sufficient conditions are verified for Lipschitz systems and a class of minimum-phase systems. Future works include the design of a pure output-feedback law, possibly by means of an incremental observer, and the use of similar strategies in more general output regulation frameworks, in which references and perturbations are generated by a known neutrally stable autonomous systems, see, e.g., [36].

\section{APPENDIX}

We review here some results and tools related to contraction theory. For a deeper analysis the reader may refer for instance to [1], [2], [4], [11], [40] and the references therein. The formal proofs of Propositions 7 and 8 are new to the author's knowledge.

\section{A. Definition and consequences of incremental exponential stability}

Consider a system of the form

$$
\dot{x}=f(x),
$$

where $x \in \mathbb{R}^{n}$ is the system's state and $f: \mathbb{R}^{n} \rightarrow \mathbb{R}^{n}$ is a $C^{1}$ vector field. Let $X\left(x_{0}, t\right)$ denote the system's trajectory evaluated at time $t$ starting from the initial condition $x_{0}$. In what follows, we don't necessary need $f(0)=0$. We have the following definition.

Definition 1 (Incremental Exponential Stability). System (54) is said to be Incrementally Exponentially Stable (in short $\delta E S)$ in a positively forward invariant set $\mathcal{C} \subseteq \mathbb{R}^{n}$ if there exists $k, \lambda \in \mathbb{R}_{>0}$ such that

$$
\left|X\left(x_{1}, t\right)-X\left(x_{2}, t\right)\right| \leq k\left|x_{1}-x_{2}\right| \exp (-\lambda t)
$$

for all $x_{1}, x_{2} \in \mathcal{C}$ and for all $t \geq 0$. In case $\mathcal{C}$ coincides with $\mathbb{R}^{n}$, we say that the system is Incrementally Globally Exponentially Stable (in short $\delta G E S$ ).

Definition 1 mainly states that any two trajectories of system (54) converge (exponentially) one to each other. Since we focused on time-invariant systems, a direct consequence of the $\delta$ GES property is stated in the following lemma (see [14. Lemma 1]).

Lemma 2 (Equilibria on Forward Invariant Sets). Suppose system [54 is $\delta E S$ in a closed forward invariant set denoted
$\mathcal{C} \subseteq \mathbb{R}^{n}$. Then, there exists a unique equilibrium point $x^{\star} \in \mathcal{C}$ which exponentially attracts all solutions initiated from $\mathcal{C}$.

Proof of Lemma 2. Let $\tau$ be such that $k \exp (-\lambda \tau)=\rho<1$ and define $\mathcal{T}_{\tau}$ as the mapping that associate to any initial condition $x_{0}$ of system of (54), its corresponding solution $X\left(x_{0}, \tau\right)$ at time $\tau$, that is $\mathcal{T}_{\tau}\left(x_{0}\right):=X\left(x_{0}, \tau\right)$. Since $\mathcal{C}$ is forward invariant, the function so defined maps points of $\mathcal{C}$ into $\mathcal{C}$. Now pick any two initial conditions $x_{1}, x_{2} \in \mathcal{C}$ and recall that system (54) is $\delta \mathrm{ES}$. Therefore, by using (55), we obtain

$$
\begin{aligned}
\left|\mathcal{T}_{\tau}\left(x_{1}\right)-\mathcal{T}_{\tau}\left(x_{2}\right)\right| & =\left|X\left(x_{1}, \tau\right)-X\left(x_{2}, \tau\right)\right| \\
& \leq k \exp (-\lambda \tau)\left|x_{1}-x_{2}\right| \leq \rho\left|x_{1}-x_{2}\right|,
\end{aligned}
$$

with $\rho<1$. Note that the Euclidean space endowed with standard Euclidean norm is a complete metric. Hence, the map $\mathcal{T}_{\tau}$ is a contraction and by Banach fixed point theorem, there exists a unique fixed point $x^{\star}$ in $\mathcal{C}$ satisfying $\mathcal{T}_{\tau}\left(x^{\star}\right)=$ $x^{\star}$. Therefore, by using again (55), we obtain

$$
\begin{aligned}
\left|X(x, t)-x^{\star}\right| & =\left|X(x, t)-X\left(x^{\star}, t\right)\right| \\
& \leq k \exp (-\lambda t)\left|x-x^{\star}\right|
\end{aligned}
$$

for any $t \geq 0$ and any $x \in \mathcal{C}$. Hence, $x^{\star}$ is attracts all trajectories initiated from $\mathcal{C}$. Finally, note that an equivalent proof of this lemma, covering also the case of periodic equilibrium, has been developed independently in [15, Section IV-B].

\section{B. A metric view point for contraction analysis}

The following result links the $\delta$ GES property of (54) with a differential analysis of the vector field $f$. In particular it is shown that the existence of a metric that decreases along the vector field guarantees incremental exponential stability of the system.

Theorem 2 (Global Uniform Contraction). Suppose there exist a $C^{1}$ function $\mathcal{P}: \mathbb{R}^{n} \rightarrow \mathbb{R}^{n \times n}$ taking positive definite symmetric values, and positive real numbers $\overline{\mathfrak{p}}, \mathfrak{p}, \mathfrak{q}>0$ satisfying

$$
\underline{\mathfrak{p}} I_{n} \leq \mathcal{P}(x) \leq \overline{\mathfrak{p}} I_{n}, \quad L_{f} \mathcal{P}(x) \leq-\mathfrak{q} I_{n},
$$

for all $x \in \mathbb{R}^{n}$. Then system (54) is $\delta G E S$ with contraction rate $\mathfrak{q}$.

Proof of Theorem 2 A proof can be found in [23, Theorem 1], [1, Section II-B], or [11].

Note that under more restrictive conditions (i.e. $f$ being $C^{2}$ with bounded first and second derivatives) it is also possible to establish a converse result, see, e.g., [1, Proposition 4]. This one is obtained since associated to the matrix function $\mathcal{P}$ we can define a Riemannian metric and its associated norm $|v|_{\mathcal{P}(\chi)}^{2}=v^{\top} \mathcal{P}(\chi) v$ on $\mathbb{R}^{n}$. The (Riemannian) distance $\mathrm{d}_{\mathcal{P}}$ between any pair of points of $\mathbb{R}^{n}$ can be defined in the following way. The length of any piecewise $C^{1}$ path $\gamma:\left[s_{a}, s_{b}\right] \rightarrow \mathbb{R}^{n}$ between two arbitrary points $\chi_{a}=\gamma\left(s_{a}\right)$ and $\chi_{b}=\gamma\left(s_{b}\right)$ in $\mathbb{R}^{n}$ is defined as

$$
\ell(\gamma)=\int_{s_{a}}^{s_{b}}\left|\frac{\partial \gamma}{\partial s}(s)\right|_{\mathcal{P}(\chi)} d s .
$$


The distance $\mathrm{d}_{\mathcal{P}}\left(\chi_{a}, \chi_{b}\right)$ is then defined as the infimum of the length over all these paths. Note that for all $\left(x_{a}, x_{b}\right)$ both in $\mathbb{R}^{n}$, inequalities (56) imply

$$
\underline{\mathfrak{p}}\left|x_{a}-x_{b}\right|^{2} \leq \mathrm{d}_{\mathcal{P}}\left(x_{a}, x_{b}\right)^{2} \leq \overline{\mathfrak{p}}\left|x_{a}-x_{b}\right|^{2} .
$$

Remark 7. The interest we have in a sufficient condition for $\delta G E S$ in the form of 56 is that this one is (almost) coordinates free. Indeed, assume that a matrix function which defines a Riemannian metric on $\mathbb{R}^{n}$ denoted $\mathcal{P}: \mathbb{R}^{n} \mapsto \mathbb{R}^{n \times n}$ is given. Let $\psi: \mathbb{R}^{n} \rightarrow \mathbb{R}^{n}$ be a global diffeomorphism. Then, the matrix function $\overline{\mathcal{P}}(z)$ defined as

$$
\overline{\mathcal{P}}(z)=\nabla \psi^{-1}(z)^{\top} \mathcal{P}\left(\psi^{-1}(z)\right) \nabla \psi^{-1}(z)
$$

satisfies $d_{\mathcal{P}}\left(x_{a}, x_{b}\right)=d_{\overline{\mathcal{P}}}\left(\psi\left(x_{a}\right), \psi\left(x_{b}\right)\right)$ for all pair $\left(x_{a}, x_{b}\right)$ in $\mathbb{R}^{n}$, where $d_{\overline{\mathcal{P}}}$ is the Riemannian distance associated to the matrix function $\overline{\mathcal{P}}$. Moreover, in the new coordinates $z:=$ $\psi(x)$, a vector fields $f: \mathbb{R}^{n} \mapsto \mathbb{R}^{n}$ becomes

$$
\bar{f}(z)=\left(\nabla \psi^{-1}(z)\right)^{-1} f\left(\psi^{-1}(z)\right)
$$

and we have, $L_{\bar{f}} \overline{\mathcal{P}}(z)=\nabla \psi^{-1}(z)^{\top} L_{f} \mathcal{P}\left(\psi^{-1}(z)\right) \nabla \psi^{-1}(z)$. Hence, if the pair $(\mathcal{P}, f)$ satisfies (56) and the diffeomorphism $\psi$ is globally Lipschitz, then the pair $(\overline{\mathcal{P}}, \bar{f})$ satisfies a similar inequality. Moreover if $f$ is a Killing vector field for $\mathcal{P}$, then so is $\bar{f}$ for $\overline{\mathcal{P}}$.

Although the result established in Theorem 2 is global, a similar result can be established whenever we restrict our attention to compact forward invariant sets. Indeed, when inequality (56) holds on the (Riemannian) convex hull of a positively invariant compact set, exponential contraction is established in this one as stated in the following proposition.

Proposition 7 (Uniform Contraction on Compact Invariant Sets). Suppose that there exist a $C^{1}$ function $\mathcal{P}: \mathbb{R}^{n} \rightarrow$ $\mathbb{R}^{n \times n}$ taking positive definite symmetric values, and positive real numbers $\overline{\mathfrak{p}}, \mathfrak{p}$, such that the first inequality in 56 holds for all $x \in \mathbb{R}^{n}$. Let $\mathcal{C}$ be a compact invariant subset of $\mathbb{R}^{n}$ for system (54) and assume that there exists $\mathfrak{q}>0$ such that inequality (56) holds for all $x \in \mathcal{O}$,

$$
\mathcal{O}:=\left\{x \in \mathbb{R}^{n}, \max _{s \in \mathcal{C}}|x-s|<\mathfrak{r}\right\},
$$

where $\mathfrak{r}>0$ is a positive real number satisfying

$$
\mathfrak{r}>\frac{\sqrt{\overline{\mathfrak{p}}}}{2 \sqrt{\mathfrak{p}}} \max _{\left(x_{a}, x_{b}\right) \in \mathcal{C}^{2}}\left|x_{a}-x_{b}\right| .
$$

Then, system [54] is $\delta E S$ on $\mathcal{C}$.

Proof of Proposition 7 Let $\mathfrak{r}_{1}$ be such that

$$
\mathfrak{r}>\mathfrak{r}_{1}>\frac{\sqrt{\mathfrak{p}}}{2 \sqrt{\mathfrak{p}}} \max _{\left(x_{a}, x_{b}\right) \in \mathcal{C}^{2}}\left|x_{a}-x_{b}\right|,
$$

and let

$$
\mathcal{O}_{1}=\left\{x \in \mathbb{R}^{n}, \max _{s \in \mathcal{C}}|x-s|<\mathfrak{r}_{1}\right\} .
$$

Note that $\mathcal{C} \subset \mathcal{O}_{1}$ and $\mathrm{Cl}\left\{\mathcal{O}_{1}\right\} \subset \mathcal{O}$.

The proof is divided into two steps. In a first step, it is shown that the (Riemannian) convex hull of $\mathcal{C}$ is included in $\mathcal{O}_{1}$ defined in $(62)$. In a second step, it is shown that the Riemannian distance between any pair or points in $\mathcal{C}$ converges exponentially to zero.

Step 1: Let $\left(x_{a}, x_{b}\right)$ be in $\mathcal{C}$. From (61), we can find $\epsilon>0$ such that

$$
\frac{\epsilon}{\sqrt{\mathfrak{p}}}<2 \mathfrak{r}_{1} \sqrt{\frac{\mathfrak{p}}{\overline{\mathfrak{p}}}}-\max _{\left(x_{a}, x_{b}\right) \in \mathcal{C}^{2}}\left|x_{a}-x_{b}\right| .
$$

We aim at showing that for all $C^{2}$ path $\gamma:\left[s_{a}, s_{b}\right] \rightarrow \mathbb{R}^{n}$ between $x_{a}=\gamma\left(s_{a}\right)$ and $x_{b}=\gamma\left(s_{b}\right)$ such that

$$
\ell(\gamma) \leq \mathrm{d}_{\mathcal{P}}\left(x_{a}, x_{b}\right)+\epsilon,
$$

this implies that $\gamma(s) \in \mathcal{O}_{1}$ for all $s \in\left[s_{a}, s_{b}\right]$. Assume the opposite. In other words, assume that there exists $s^{*}$ in $\left[s_{a}, s_{b}\right]$ such that $\gamma\left(s^{*}\right) \notin \mathcal{O}_{1}$. With [62), this implies that $\left|\gamma\left(s^{*}\right)-x_{a}\right| \geq \mathfrak{r}_{1}$ and $\left|\gamma\left(s^{*}\right)-x_{b}\right| \geq \mathfrak{r}_{1}$. Consequently, in combination with the left-hand side of (58), it implies

$$
\begin{aligned}
\ell(\gamma) & \geq \mathrm{d}_{\mathcal{P}}\left(x_{a}, \gamma\left(s^{*}\right)\right)+\mathrm{d}_{\mathcal{P}}\left(x_{b}, \gamma\left(s^{*}\right)\right) \\
& \geq \sqrt{\underline{\mathfrak{p}}}\left(\left|x_{a}-\gamma\left(s^{*}\right)\right|+\left|x_{b}-\gamma\left(s^{*}\right)\right|\right) \geq 2 \mathfrak{r}_{1} \sqrt{\underline{\mathfrak{p}}} .
\end{aligned}
$$

On the other hand, with 64 , the right-hand side of (58) and 63, it yields

$$
\ell(\gamma) \leq \sqrt{\overline{\mathfrak{p}}}\left|x_{a}-x_{b}\right|+\epsilon<2 \mathfrak{r}_{1} \sqrt{\underline{\mathfrak{p}}} .
$$

This yields a contradiction. Consequently, for all $C^{2}$ paths such that (64) holds, $\gamma(\cdot)$ takes values in $\mathcal{O}_{1}$.

Step 2: Let now $T>0$ be a real number such that

$$
X(x, t) \in \mathcal{O}, \quad \forall(x, t) \in \mathcal{O}_{1} \times[0, T] .
$$

Such a $T$ exists since there exists a minimal distance between the boundary of the two sets, and $\mathcal{O}$ is bounded. Consider a $C^{2}$ path between $\left(x_{a}, x_{b}\right) \in \mathcal{C}^{2}$ satisfying (64). With Step 1, we know that $\gamma:\left[s_{a}, s_{b}\right] \rightarrow \mathcal{O}_{1}$. For all $(s, t) \in\left[s_{a}, s_{b}\right] \times$ $[0, T]$ we can define

$$
\Gamma(s, t):=X(\gamma(s), t), \quad \rho(s, t):=\left|\frac{\partial \Gamma}{\partial s}(s, t)\right|_{\mathcal{P}(\Gamma(s, t))}^{2} .
$$

Due to 67], $\Gamma(s, t) \in \mathcal{O}$ for all $(s, t) \in\left[s_{a}, s_{b}\right] \times[0, T]$. Then $\rho$ is $C^{1}$ and equation 56 yields

$$
\begin{aligned}
\frac{\partial \rho}{\partial t}(s, t) & =\frac{\partial \Gamma^{\top}}{\partial s}(s, t) L_{f} \mathcal{P}(\Gamma(s, t)) \frac{\partial \Gamma}{\partial s}(s, t) \\
& \leq-\mathfrak{q}\left|\frac{\partial \Gamma}{\partial s}(s, t)\right|^{2} \leq-\frac{\mathfrak{q}}{\overline{\mathfrak{p}}} \rho(s, t),
\end{aligned}
$$

which yields

$$
\begin{aligned}
\frac{d \ell(\Gamma(\cdot, t))}{d t} & =\frac{d}{d t} \int_{s_{a}}^{s_{b}} \sqrt{\rho(s, t)} d s \\
& =\int_{s_{a}}^{s_{b}} \frac{1}{2 \sqrt{\rho(s, t)}} \frac{\partial \rho}{\partial t}(s, t) d s \leq-\frac{\mathfrak{q}}{2 \overline{\mathfrak{p}}} \ell(\Gamma(\cdot, t)) .
\end{aligned}
$$

Hence $\ell(\Gamma(\cdot, T)) \leq \ell(\gamma) \exp \left(-\frac{\mathfrak{q}}{2 \bar{p}} T\right)$. Choosing a sequence of paths $\left(\gamma_{n}\right)_{n \in \mathbb{N}}$ such that $\ell\left(\gamma_{n}\right) \rightarrow \mathrm{d}_{\mathcal{P}}\left(x_{a}, x_{b}\right)$ and satisfying (64) and passing to the limit, we obtain

$$
\mathrm{d}_{\mathcal{P}}\left(X\left(x_{a}, T\right), X\left(x_{b}, T\right)\right)<\kappa \mathrm{d}_{\mathcal{P}}\left(x_{a}, x_{b}\right) .
$$


for some $0<\kappa<1$. This property is true for all $\left(x_{a}, x_{b}\right)$ in $\mathcal{C}^{2}$. Recalling the positive invariance of $\mathcal{C}$, it implies that (55) holds.

Proposition 8 (Non-uniform Global Contraction). Suppose there exist a $C^{1}$ function $\mathcal{P}: \mathbb{R}^{n} \rightarrow \mathbb{R}^{n \times n}$ taking positive definite symmetric values, a $C^{0}$ function $\mathfrak{q}: \mathbb{R}^{n} \rightarrow \mathbb{R}_{>0}$ and positive real numbers $\overline{\mathfrak{p}}, \mathfrak{p}>0$, such that, for all $x \in \mathbb{R}^{n}$, the following properties hold

$$
\underline{\mathfrak{p}} I_{n} \leq \mathcal{P}(x) \leq \overline{\mathfrak{p}} I_{n}, \quad L_{f} \mathcal{P}(x) \leq-\mathfrak{q}(x) I_{n}<0 .
$$

Then, there exists $k \in \mathbb{R}_{>0}$ such that

$$
\left|X\left(x_{a}, t\right)-X\left(x_{b}, t\right)\right| \leq k\left|x_{a}-x_{b}\right|
$$

for all $\left(x_{a}, x_{b}\right)$ in $\mathbb{R}^{n} \times \mathbb{R}^{n}$ and for all $t \geq 0$. Moreover, if there exists an equilibrium point $x^{\star} \in \mathbb{R}^{n}$, then $x^{\star}$ is unique and it is globally attractive for system (54).

Proof of Proposition 8, Since system (54) satisfies (69), following the same steps that in Step 2 of the proof of Proposition 7 it can be shown that picking any two points $\left(x_{a}, x_{b}\right)$ in $\mathbb{R}^{n} \times \mathbb{R}^{n}$, it yields $\mathrm{d}_{\mathcal{P}}\left(X\left(x_{a}, t\right), X\left(x_{b}, t\right)\right) \leq \mathrm{d}_{\mathcal{P}}\left(x_{a}, x_{b}\right)$. Employing equation (58), inequality (70) holds. In the particular case in which there exists an equilibrium $x^{*}$, then for all $x$ in $\mathbb{R}^{n}$ (70) yields $\mathrm{d}_{\mathcal{P}}\left(X(x, t), x^{*}\right) \leq \mathrm{d}_{\mathcal{P}}\left(x, x^{*}\right)$. Hence, for all $\bar{d} \geq 0$, the set $\mathcal{C}:=\operatorname{Cl}\left\{x, \mathrm{~d}_{\mathcal{P}}\left(x, x^{*}\right) \leq \bar{d}\right\}$ is a compact invariant subset of $\mathbb{R}^{n}$. Let $\underline{\mathfrak{q}}_{\mathcal{O}}:=\min _{\{x \in \mathcal{O}\}} \mathfrak{q}(x)>0$, where $\mathcal{O}:=\left\{x \in \mathbb{R}^{n}, \max _{s \in \mathcal{C}}|x-s|<\mathfrak{r},\right\}$, for some positive real number $\mathfrak{r}$ satisfying $\mathfrak{r}>\frac{\sqrt{\mathfrak{p}}}{2 \sqrt{\mathfrak{p}}} \max _{\left(x_{1}, x_{2}\right) \in \mathcal{C}^{2}}\left|x_{1}-x_{2}\right|$. Hence, in view of Proposition 7 this implies that the system (54) is $\delta \mathrm{ES}$ on $\mathcal{C}$, and application of Lemma 2 implies the existence of a unique equilibrium which is attractive from $\mathcal{C}$. Since these arguments holds for any $\bar{d} \geq 0$, we conclude that $x^{\star}$ is the unique equilibrium globally attractive.

Example. As a simple example of application of Propositions 7 and 8 , consider the following scalar system

$$
\dot{x}=f_{w}(x)=-\arctan (x)+w, \quad x \in \mathbb{R},
$$

where the function $f_{w}$ is parametrized by $w \in \mathbb{R}$. The vector field associated to system (71) is not a strictly contraction. Indeed, Indeed, by selecting $P(x)=1$, we obtain

$$
L_{f} \mathcal{P}(x)=-\left(1+x^{2}\right)^{-1}:=-\mathfrak{q}(x)<0 \quad \forall x \in \mathbb{R} .
$$

Since $\lim _{|x| \rightarrow \infty} \mathfrak{q}(x)=0$, inequality (56) is not verified, although 69 does. Nonetheless, given any compact set $\mathcal{C}=\{|x| \leq c\}, c>0$, inequality (56) is verified on $\mathcal{C}$ with $\mathfrak{q}:=\frac{1}{1+c^{2}}$. Hence, system (71) is $\delta \mathrm{ES}$ on any compact forward invariant set $\mathcal{C}$, according to Proposition 7 . Furthermore, for any $w$ satisfying $|w|<\frac{\pi}{2}$, system 71] admits an equilibrium $x^{\star}=\tan (w)$ which is globally attractive by Proposition 8 .

\section{REFERENCES}

[1] V. Andrieu, B. Jayawardhana, and L. Praly. Transverse exponential stability and applications. IEEE Transactions on Automatic Control, 61(11):3396-3411, 2016

[2] V. Andrieu, B. Jayawardhana, and S. Tarbouriech. Some results on exponential synchronization of nonlinear systems. IEEE Transactions on Automatic Control, 63(4):1213-1219, 2018.

[3] V. Andrieu and S. Tarbouriech. LMI conditions for contraction and synchronization. IFAC-PapersOnLine, 52(16):616-621, 2019.

[4] D. Angeli. A lyapunov approach to incremental stability properties. IEEE Transactions on Automatic Control, 47(3):410-421, 2002.

[5] D. Astolfi and L. Praly. Integral action in output feedback for multiinput multi-output nonlinear systems. IEEE Transactions on Automatic Control, 62(4):1559-1574, 2017.

[6] S. Behtash. Robust output tracking for non-linear systems. International Journal of Control, 51(6):1381-1407, 1990.

[7] M. Bin and L. Marconi. Output regulation by postprocessing internal models for a class of multivariable nonlinear systems. International Journal of Robust and Nonlinear Control, 30(3):1115-1140, 2020.

[8] L. D‘Alto and M. Corless. Incremental quadratic stability. Numerical Algebra, Control and Optimization, 3(1):175-201, 2013.

[9] E. J. Davison. The robust control of a servomechanism problem for linear time-invariant multivariable systems. IEEE Trans. Autom. Contr., AC-21(1):25-34, 1976.

[10] C. Desoer and CA. Lin. Tracking and disturbance rejection of mimo nonlinear systems with pi controller. IEEE Transactions on Automatic Control, 30(9):861-867, 1985.

[11] F. Forni and R. Sepulchre. A differential lyapunov framework for contraction analysis. IEEE Transactions on Automatic Control, 59(3):614628, 2013.

[12] B.A. Francis and W.M. Wonham. The internal model principle of control theory. Automatica, 12(5):457-465, 1976.

[13] M. Giaccagli, V. Andrieu, D. Astolfi, and G. Casadei. Sufficient metric conditions for synchronization of leader-connected homogeneous nonlinear multi-agent systems. IFAC-PapersOnLine, 54(14):412-417, 2021.

[14] M. Giaccagli, D. Astolfi, V. Andrieu, and L. Marconi. Sufficient conditions for output reference tracking for nonlinear systems: a contractive approach. In $59^{\text {th }}$ IEEE Conference on Decision and Control, 2020.

[15] W.P.M.H. Heemels, M.K. Camlibel, and M.F. Heertjes. Oblique projected dynamical systems and incremental stability under state constraints. IEEE Control Systems Letters, 2020.

[16] X. Huang, H.K Khalil, and Y. Song. Regulation of nonminimum-phase nonlinear systems using slow integrators and high-gain feedback. IEEE Transactions on Automatic Control, 64(2):640-653, 2018.

[17] A. Isidori. Nonlinear Control Systems. Springer, 1995.

[18] A. Isidori, L. Marconi, and A. Serrani. Robust autonomous guidance: an internal model approach. Springer Science \& Business Media, 2012.

[19] ZP. Jiang and I. Marcels. Robust nonlinear integral control. IEEE Transactions on Automatic Control, 46(8):1336-1342, 2001.

[20] G. Kaliora and A. Astolfi. Nonlinear control of feedforward systems with bounded signals. IEEE Transactions on Automatic Control, 49(11):1975-1990, 2004.

[21] H.K Khalil. Universal integral controllers for minimum-phase nonlinear systems. IEEE Transactions on Automatic Control, 45(3):490-494, 2000.

[22] H.K. Khalil. Nonlinear systems, volume 3. Prentice hall, 2002.

[23] DC Lewis. Metric properties of differential equations. American Journal of Mathematics, 71(2):294-312, 1949.

[24] N. A Mahmoud and H. K Khalil. Asymptotic regulation of minimum phase nonlinear systems using output feedback. IEEE Transactions on Automatic Control, 41(10):1402-1412, 1996.

[25] I.R. Manchester and JJ. Slotine. Control contraction metrics: Convex and intrinsic criteria for nonlinear feedback design. IEEE Transactions on Automatic Control, 62(6):3046-3053, 2017.

[26] R. Marino and P. Tomei. Output regulation for linear minimum phase systems with unknown order exosystem. IEEE Transactions on Automatic Control, 52(10):2000-2005, 2007.

[27] F. Mazenc and L. Praly. Adding integrations, saturated controls, and stabilization for feedforward systems. IEEE Transactions on Automatic Control, 41(11):1559-1578, 1996.

[28] K. Nomizu. On local and global existence of killing vector fields. Annals of Mathematics, pages 105-120, 1960. 
[29] A. Pavlov and L. Marconi. Incremental passivity and output regulation. Systems \& Control Letters, 57(5):400-409, 2008.

[30] A. Pavlov, E. Steur, and N. van de Wouw. Controlled synchronization via nonlinear integral coupling. In 48 IEEE Conference on Decision and Control, pages 5263-5268, 2009.

[31] A. Pavlov, N. van de Wouw, and H. Nijmeijer. Uniform output regulation of nonlinear systems: a convergent dynamics approach. Springer Science \& Business Media, 2006.

[32] F. Poulain and L. Praly. Robust asymptotic stabilization of nonlinear systems by state feedback. IFAC Proceedings Volumes, 43(14):653658, 2010.

[33] L. Praly, R. Ortega, and G. Kaliora. Stabilization of nonlinear systems via forwarding mod $\left\{L_{g} V\right\}$. IEEE Transactions on Automatic Control, 46(9):1461-1466, 2001

[34] L. Praly and Y. Wang. Stabilization in spite of matched unmodeled dynamics and an equivalent definition of input-to-state stability. Mathematics of Control, Signals and Systems, 9(1):1-33, 1996.

[35] Zhihua Qu. Robust control of nonlinear uncertain systems under generalized matching conditions. Automatica, 29(4):985-998, 1993.

[36] A. Serrani, A. Isidori, and L. Marconi. Semi-global nonlinear output regulation with adaptive internal model. IEEE Transactions on Automatic Control, 46(8):1178-1194, 2001.

[37] S. Seshagiri and H. K Khalil. Robust output feedback regulation of minimum-phase nonlinear systems using conditional integrators. Automatica, 41(1):43-54, 2005.

[38] T. Simon, M. Giaccagli, J.F. Trégouët, D. Astolfi, V. Andrieu, X. LinShi, and H. Morel. Robust output set-point tracking for a power flow controller via forwarding design. In 2021 60th IEEE Conference on Decision and Control (CDC), 2021

[39] J.W. Simpson-Porco. Analysis and synthesis of low-gain integral controllers for nonlinear systems with application to feedback-based optimization. arXiv preprint arXiv:2003.01348, 2020.

[40] J.W Simpson-Porco and F. Bullo. Contraction theory on riemannian manifolds. Systems \& Control Letters, 65:74-80, 2014.

[41] G. Teschl. Ordinary differential equations and dynamical systems, volume 140. American Mathematical Soc., 2012.

[42] H. Tsukamoto, S.J. Chung, and J.J. E. Slotine. Contraction theory for nonlinear stability analysis and learning-based control: A tutorial overview. Annual Reviews in Control, 2021.

[43] S. Waitman, L. Bako, P. Massioni, G. Scorletti, and V. Fromion. Incremental stability of lur'e systems through piecewise-affine approximations. IFAC-PapersOnLine, 50(1):1673-1679, 2017.

[44] L. Wang, L. Marconi, C. Wen, and H. Su. Pre-processing nonlinear output regulation with non-vanishing measurements. Automatica, 111:108616, 2020.

[45] VA Yakubovich. Matrix inequalities method in stability theory for nonlinear control systems: I. absolute stability of forced vibrations. Automation and remote control, 7(905-917):202, 1964.

[46] M. Zamani, N. van de Wouw, and R. Majumdar. Backstepping controller synthesis and characterizations of incremental stability. Systems \& Control Letters, 62(10):949-962, 2013.

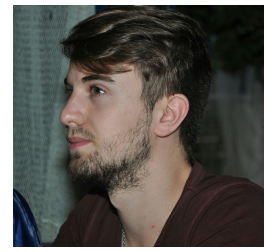

Mattia Giaccagli was born in 1995. Received the B.S. and M.S. degrees with honors in automation engineering from the University of Bologna, Italy, in 2017 and 2019, respectively. He is currently a Ph.D. candidate at LAGEPP-CNRS, Université de Lyon 1, France. His research topics include incremental stability theory and output regulation.

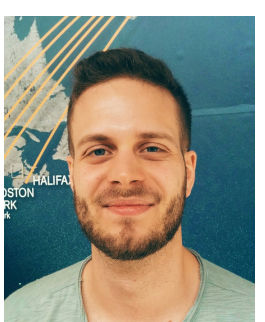

Daniele Astolfi received the B.S. and M.S. degrees in automation engineering from the University of Bologna, Italy, in 2009 and 2012, respectively. He obtained a joint Ph.D. degree in Control Theory from the University of Bologna, Italy, and from Mines ParisTech, France, in 2016. In 2016 and 2017, he has been a Research Assistant at the University of Lorraine (CRAN), Nancy, France. Since 2018, he is a CNRS Researcher at LAGEPP, Lyon, France. His research interests include observer design, feedback stabilization and output regulation for nonlinear systems, networked control systems, hybrid systems, and multi-agent systems. He serves as an associate editor of the IFAC journal Automatica. He was a recipient of the 2016 Best Italian Ph.D. Thesis Award in Automatica given by SIDRA.

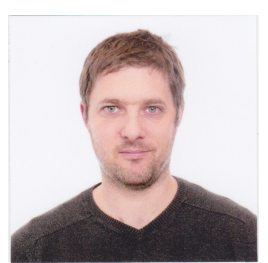

Vincent Andrieu is a Senior Reseacher at CNRS (Directeur de recherche). He graduated in applied mathematics from INSA de Rouen, France, in 2001. After working in ONERA (French aerospace research company), he obtained a $\mathrm{PhD}$ degree from Ecole des Mines de Paris in 2005. In 2006, he had a research appointment at the Control and Power Group, Dept. EEE, Imperial College London. In 2008, he joined the CNRS-LAAS lab in Toulouse, France, as a CNRS-charge de recherche. Since 2010, he has been working in LAGEP-CNRS, Universite de Lyon 1, France. In 2014, he joined the functional analysis group from Bergische Universitat Wuppertal in Germany, for two sabbatical years. His main research interests are in the feedback stabilization of controlled dynamical nonlinear systems and state estimation problems. He is also interested in practical application of these theoretical problems, and especially in the field of aeronautics and chemical engineering. Since 2018 he is an associate editor of the IEEE Transactions on Automatic Control, and senior editor for System \& Control Letters.

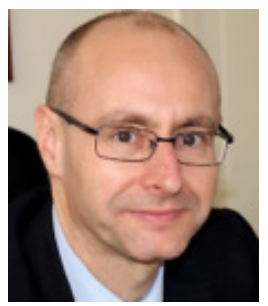

Lorenzo Marconi graduated in 1995 in electrical engineering from the University of Bologna. Since 1995 he has been with the Department of Electronics, Computer Science and Systems at University of Bologna, where he obtained his Ph.D. degree in 1998. From 1999 he has been an assistant professor in the same department where he is now a full professor. He has held visiting positions at various academic/research international institutions. He is a co-author of more than 250 technical publications on the subject of linear and nonlinear feedback designs published on international journals, books and conference proceedings. In 2005, he received the "Outstanding Application Paper Award" from IFAC for a coauthored paper published on Automatica. He is also the co-recipient of the 2014 IEEE Control Systems Magazine Outstanding Paper Award for the best paper published in the magazine in the period 2012-2013 and of the 2018 O. Hugo Schuck Best Paper Award. He is Fellow of IEEE for contributions to feedback design of nonlinear systems and unmanned aerial vehicles. His current research interests include nonlinear control, output regulation, control of autonomous vehicles, fault detection and isolation, and fault tolerant control. 